\title{
Dendrimeric Biocides - A Tool for Effective Antimicrobial Therapy
}

Karthikeyan $\mathbf{R}^{1 *}$, Kumar $\mathbf{P V}^{2}$ and Koushik OS $^{1}$

${ }^{1}$ Vignan Pharmacy College, vadlamudi, AP, India

${ }^{2}$ Menara Gading, UCSI University (South Campus), Taman Connaught, Cheras, Kuala Lumpur, Malaysia

\begin{abstract}
Agents that are capable of killing pathogenic microorganisms are known as Antimicrobial Substances. These are of low molecular weight and used for the sterilization of water, as antimicrobial drugs, as food preservatives, and for soil sterilization. Due to their potential to provide quality and safety benefits to many materials antimicrobials gain interest from both industry and academic research. However, antimicrobial agents with low molecular weight suffer from many disadvantages, such as the antimicrobial ability to short-term and environment toxicity. Antimicrobial functional groups can be introduced into polymer molecules to overcome problems associated with the low molecular weight antimicrobial agents. For enhancing the efficacy of some existing antimicrobial agents, selectivity, increasing their efficiency and prolonging the lifetime of the antimicrobial agents the use of antimicrobial polymers can be employed. The development of antimicrobial polymers research represents a great a challenge for both the academic world and industry. The polymer research that presents great modern interest, yet has received lacking consideration, is that of the development of polymers with antimicrobial activities, commonly known as polymeric biocides. Biocides immobilized on dendrimers can be more effective if the target sites are cell walls or membranes. It has been shown that small quaternary ammonium compounds exert their antimicrobial action by disintegrating and disrupting the cell membrane, converting functional end groups of dendrimer to ammonium salts, dendrimer biocides can be synthesized. These dendrimer biocides have been shown to be more potent than their small molecule counterparts as they bear high local density active groups. Thus, dendrimer biocides may be very beneficial in terms of activity, reduced toxicity, localization in specific organs and increased duration of action. This chapter will find complete solution for an antimicrobial agent facing problems and advantages of the dendrimeric biocides.
\end{abstract}

Keywords: Dendrimers; Antibacterial therapy; Solubility enhancement; Resistance strain targeting; Phagocytosis enhancement

\section{Introduction}

\section{Bacterial infection}

Bacteria are common infective agents producing a wide variety of diseases. In developing countries and the origin of massive epidemics bacteria is still an important cause of mortality that spread very easily among the population of developing countries due to the lack of adequate sanitary conditions. Additionally, some bacteria are responsible to produce highly potent toxins that can be considered as biological warfare. The search for therapeutic and prophylactic agents against these toxins is a topic of extreme importance [1].

The appearance of bacterial resistance is due to the broad use and sometimes abuse of antibiotics that has forced the biomedical researchers to look for new strategies to combat bacterial infections. One of the most attractive alternatives is using antiadhesive molecules in the inhibition of bacterial attachment to target cells during the first stages of the infection. Bacterial adhesion is mediated between lectinlike proteins by specific interactions of carbohydrate-protein at the surface of bacteria and glycoconjugates at the surface of target cells or viceversa. Blocking this interaction should inhibit the attachment of bacteria to the target cell surface and stop the infection. Due to the presence of multivalent interactions the efficiency of this recognition process is achieved in nature, even though carbohydrate-protein interactions are weak. Based on the type of bacterial target the classification of many different structures are described.

\section{Bacteria producing $\mathrm{AB}_{5}$ toxins}

Gram-negative bacteria are very important group causing thousands of deaths every year by producing toxins. Generally these toxins consists of six subunits, subunit ' $A$ ' that is responsible of the infection and a homopentameric subunits ' $\mathrm{B}$ ' $\left(\mathrm{B}_{5}\right)$ that are required for the toxin to attach to the cell surface. Inhibition of the attachment of the subunits $B_{5}$ should be enough to stop the infection process. In a multivalent way this $B$ unit has a carbohydrate recognition site that interacts with carbohydrates present at the cell surface.

\section{Cholera toxin}

Cholera toxin is an $\mathrm{AB}_{5}$ protein secreted by Vibrio cholerae causing the $\mathrm{c}$ cholera. This is an infectious intestinal disease characterized by vomiting and severe diarrhea, that if untreated may be life-threatening due to enormous loss of water and electrolytes. The B subunit is able to recognize and interact with ganglioside GM1 at the cell membrane forming a pore for subunit A that blocks GTPase activity of G protein and results in an increase of the synthesis of cAMP. As $\mathrm{Cl}^{-}$leaves the cells followed by $\mathrm{Na}^{+}$and water leads to watery, electrolyte rich diarrhea in the intestine.

\section{Shiga and vero toxins}

Shiga toxins produced by Shigella dysenteriae and Shiga-like toxins also called Vero toxins produced by Escherichia coli are $\mathrm{AB}_{5}$ toxins causing hemorrhagic colitis and watery diarrhea particularly severe in children and elder people.

*Corresponding author: Karthikeyan R, Oruganti Sai Koushik, Vignan Pharmacy College, Vadlamudi-522213. A.P. India, Tel: 919966847127; E-mail: rkcognosy@gmail.com

Received February 01, 2016; Accepted February 15, 2016; Published February 25, 2016

Citation: Karthikeyan R, Kumar PV, Koushik OS (2016) Dendrimeric Biocides - A Tool for Effective Antimicrobial Therapy. J Nanomed Nanotechnol 7: 359. doi:10.4172/2157-7439.1000359

Copyright: $\odot 2016$ Karthikeyan R, et al. This is an open-access article distributed under the terms of the Creative Commons Attribution License, which permits unrestricted use, distribution, and reproduction in any medium, provided the original author and source are credited. 


\section{Heat labile enterotoxin}

The heat labile enterotoxin of $E$. coli is a cholera-like enterotoxin. It adheres as cholera toxin does to ganglioside GM1 and causes less severe diarrhea due to the same mechanisms [2]. The group of Schengrund has used the same type of experiments to show the activity of oligosaccharide-derivatized dendrimers for cholera toxin and heat labile toxin with similar results based on the structural similarities between cholera toxin and heat labile toxin [3].

\section{Bacterial endotoxins}

The lipopolysaccharide (LPS) consists of a bilayered component membrane which plays a key role in septic shock present at the outer leaflet of Gram-negative bacteria such as Haemophilus influenzae, Kelbsiella pneumoniae, Bordetellapertussis, Pseudomonas aeruginosa, Chlamydia psittaci, Escherichia coli, Salmonella enterica and Legionella pneumophila [4]. When bacteria multiply, die or lyse this LPS is liberated from the membrane surface. In many cases of death they have been recognized LPS as a factor, responsible of toxicity in severe Gram-negative bacteria infections. It causes a systemic response that if uncontrolled can lead to septic shock characterized mainly by hypotension, coagulopathy, fever, and organ failure. Lipid A is a glycolipid toxic moiety of LPS considered as a target for the design of drugs against endotoxins. This LPS presents an anionic and amphiphilic nature that is an important feature for the design of compounds able to interact with LPS.

\section{Type 1 fimbriated Escherichia coli}

Most of the Gram-negative bacteria possess type 1 fimbriae, which are responsible for the adherence of Escherichia coli to the urinary tract by adhesion organelles causes common urinary tract infection [5]. This adhesion process is governed by the interaction between type 1 fimbriae and mannose conjugates found at the bladder epithelial cell surface.

\section{Streptococcus suis}

Gram-positive bacteria like Streptococcus suis is responsible for septicemia, meningitis and pneumonia in swine, pigs and other domestic animals. It is responsible for meningitis in humans when being in contact with pigs [6]. In the adhesion process of the bacteria to the host cells this bacteria contains a galactosyl-1-4-galactose-binding adhesin [7].

\section{Staphylococcus aureus}

In 1880 and 1882 Staphylococcal disease and its role in sepsis and abscess formation were described for the first time by Ogston. More than 100 years later, Staphylococcus aureus remains a dangerous pathogen in humans that can cause illeffects from minor skin infections to life-threatening diseases [8].

\section{Actinomyces naeslundii}

A. naeslundii is a Gram-positive bacterium that colonizesoral cavities. During this colonization process, Streptococcus oralis coaggregate with $A$. naeslundii through galactose residues present at the surface of $S$. oralis and an adhesin of A. naeslundii pili [9].

\section{Fungal infection}

Candida albicans causes most common infections produced by fungi. Under normal circumstances, $C$. albicans colonizes humans without any harmful effects, although excess may result in candidiasis in skin or mucosa [10]. Systemic candidiasis is often observed in immunocompromised individuals. At the surface of target cells different forms of C. albicans are also recognized by different lectins [11].

\section{Prion protein infection}

Almost 25 years ago Stanley Prusiner discovered prion a new infective agent and protein. The prion protein is the product of a normal gene expressed mainly in neural tissue and has several helixes of few sheets in its natural configuration known as $\operatorname{PrP}^{\mathrm{C}}$. This protein adopts an abnormal configuration upon contact with the sheet rich infectious form of the protein known as $\mathrm{PrP}^{\mathrm{Sc}}$ after scrapie, an old recognized disease of sheep [12]. These types of structures are found in several neurodegenerative disorders such as Creutzfeldt-Jakob disease in humans, bovine spongiform encephalopathy etc.

\section{Viral infection}

This is the area of application where more efforts have been done respect to develop new anti-infective agents based on dendrimers. Recently, a review describing dendrimers as antivirals has been published [13]. Here, we intend to update the information presented in that review with the most recent publications concerning dendrimers as antiviral drugs. Again, this section will be divided for each different viral agent.

\section{HIV-1, HIV-2, and SIV}

Infection by Human Immunodeficiency Virus (HIV) is a global health problem although, especially dramatic in developing countries in sub-Saharan Africa and Asia where the vast majority of infected patients do not have access to antiretroviral drugs. Recent research in this topic is concentrated mainly on developing microbicides and vaccine development [14].

\section{Herpes simplex virus (HSV) infection}

One of the most prevalent sexual transmitted diseases is Genital human herpes virus infection. HSV-1 and 2 cause mucocutaneous infection, such as herpes labialis and herpes genitalis. Currently, no cure is available, in neurons of the host after primary or initial infection the virus persists for life in a latent form, periodically reactivating. [15].

\section{Influenza virus infection}

Influenza virus is a RNA virus. They are 3 types of influenza: A, $\mathrm{B}$, and C. Influenza A is the cause of all flu pandemics. Through the interaction of the main envelope glycoprotein: hemagglutinin (HA) this virus adheres to the target cells. HA recognizes sialic acid receptors on the host cell. It is known that monovalent sialic acid was able to prevent influenza A agglutination of chicken erythrocytes [16].

\section{Food-and-mouth disease virus (FMDV) infection}

In farms virus infects animals through the respiratory tract or skin abrasions [17]. To generate a vaccine against this infection a very interesting approach has been described by Andreu et al. [18]

\section{Ebola virus infection}

The Filoviridae family that is responsible of sporadic outbreaks of hemorrhagic fever in Africa characterized by a high death rate [19]. There is not currently any vaccine or specific treatment available for these dangerous agents and only supportive measures can be provided for infected individuals. The envelope of Ebola virus consists of a trimer, highly glycosilated glycoprotein that is recognized both by DC-SIGN 
and DC-SIGNR/L-SIGN. It has been shown invitro that the presence of these molecules can significantly increase the infectivity facilitating entry in cis and in trans, i.e. to susceptible neighbouring cells [20].

\section{Problems Associated with Conventional Antimicrobial Therapy}

Conventional antimicrobial therapy consists of chemotherapeutic agents to treat the infectious diseases by either killing of the microbes, or interfering with their growth. With the commercial production of the first antibiotic penicillin in the late 1940s, use of the antibiotics to treat the infectious diseases increased and to-date many new antibiotics have been developed [21], ranging from the topical antibiotic ointments to intravenously injected antibiotic solutions. These drugs have proven to be effective in eliminating the microbial infections that arise from minor cuts and scrapes to life threatening infections. An antimicrobial drugs act on the microbes by various mechanisms such as inhibiting cell wall synthesis, inhibiting the protein synthesis, inhibiting the nucleic acid synthesis, inhibiting the metabolic pathways, and by interfering with the membrane integrity [22]. Being a lifesaving drug for so many decades, antibiotics do suffer from a range of limitations, which include narrow spectrum of antimicrobial activity, problem regarding the safety and tolerability of the antimicrobial agent, antibiotic mediated enhancement of microbial virulence properties which may also lead to prolongation of host carrier state and may lead to harmful side effect to the host such as toxicity, or any allergic reaction. Inefficient delivery of the drugs has also been one of the major limitations of conventional antimicrobial therapy.

Another major limitation of antimicrobial therapy is the development of bacterial resistance to antibiotics. More than $70 \%$ of bacteria causing infections are now resistant to at least one of the drugs most commonly used for the treatment. Some organisms are so reluctant that they can only be treated with the experimental and potentially toxic drugs. These microbes use diverse mechanisms to develop the resistance against the antibiotics such as they may alter the drug target, inactivate enzymes, inhibit efflux transport, or develop alternate metabolic pathways for their growth. Some of the important resistant bacteria along with their resistance mechanisms are listed in Table 1. One of the serious clinical threats in treating the infections via antibiotics emerged with the development of vancomycin-resistant Enterococcus (VRE) which showed resistance to many commonly used antibiotics [23]. Another example is that of methicillin resistant Staphylococcus aureus (MRSA) strains that have caused great concern due to potential spread of antibiotic resistance. Cohen (2000) reported that more than $40 \%$ of $S$. aureus strains collected from the hospitals weremethicillin resistant and some of them were resistant to vancomycin. One of the global and medical challenges in the $21^{\text {st }}$ century is the treatment of vancomycin-resistant microbes because vancomycin is the latest generation of antibiotics and assumed most effective for S. aureus infection [24]. Problems with multiple drug resistance are also increasing in nosocomial Gram-negative bacteria, which have the capability of developing different mechanisms for antibiotic resistance

The chemotherapy of infections caused by bacteria that inhabit intracellularly presents a number of uncommon challenges. Many bacteria have found the way to produce a "silent" infection inside the cells and to avoid from their bactericidal mechanisms. There is an essential need for new and improved approaches for bacterial destruction. Although the therapeutic efficacy of drugs has been well recognized, inefficient delivery could result in insufficient therapeutic index. It is now clear that a nanotechnology-driven approach using nanoparticles to selectively target and destroy pathogenic bacteria can be successfully implemented. Nanotechnology is one approach to overcome challenges of conventional drug delivery systems based on the development and fabrication of nanostructures. Some challenges associated with the technology are as it relates to drug effectiveness, toxicity, stability, and pharmacokinetics and drug regulatory control. Localized diseases such as infection and inflammation not only have perforated vasculature but also overexpress some epitopes or receptors that can be used as targets. Thus, nanomedicines can also be actively targeted to these locations. Various types of nanoparticulate systems have been tried as potential drug delivery systems, containing biodegradable polymeric nanoparticles, polymeric micelles, nanocap- sules, nanogels, fullerenes, solid lipid nanoparticles (SLN), nanoliposomes, dendrimers, metal nanoparticles and quantum dots. Nanoparticles have been found useful in the development of systemic, oral, pulmonary, transdermal and other administration routes to study drug targeting, the enhancement of drug bioavailability and protection of drug bioactivity and stability. In recent years, encapsulation of antimicrobial drugs in nanoparticle systems has emerged as an innovative and promising alternative that enhances therapeutic effectiveness and minimizes the undesirable side effects of drugs. The major goals in designing nanoparticles as delivery systems are to control particle size, surface properties and release of pharmacologically active agents in order to achieve the sitespecific action at the therapeutically optimal rate and dose regimen. This chapter focuses on nanoparticle-based drug delivery systems and clinical applications to treat a variety of bacterial infectious diseases and their potential applications in the field of medicine and biology.

\section{Types of infections}

Infectious disease is a clinically obvious disorder resulting from the presence of a pathogenic agent which can either be a virus, bacterium, fungus or parasite. These diseases are also called communicable diseases due to their ability to get transferred from one person to another (malaria, tuberculosis) and also sometimes from one species to another (flu, influenza). Infectious diseases can be vastly classified as: 1) known diseases which are insistently there (e.g., dengue, malaria, tuberculosis); 2) new, previously unknown diseases (e.g., severe acute respiratory syndrome); and 3) diseases which threaten to enhance in

\begin{tabular}{|c|c|c|}
\hline Drug & Microorganism & Mechanism of resistance \\
\hline$\beta$-lactum (carbapen) & Enterobactericeae (E. coli) & Drug degrading enzyme \\
\hline$\beta$-lactum (methicillin) & Staphylococcus aureus & Production of an additional enzyme that avoids binding \\
\hline Macrolide & Streptococcus pneumoniae & Changes in target \\
\hline Vancomycin & Enterococcus & Cell wall thickening changes in target \\
\hline Multiple drugs & Pseudomonas aeruginosa & Mutation in target \\
\hline Vancomycin & Staphylococcus aureus & Over production of target site \\
\hline Quinolone & Gonococci & Enterococcus \\
\hline Sulfonamide & & Ong \\
\hline
\end{tabular}

Table 1: The list of drug resistant bacteria along with their mechanism of resistance. 
the near future (e.g., avian influenza). These diseases own a great risk as more than half of the deaths happening world- wide can be attributed to these diseases, particularly in developing countries [25]. Parasitism is based on the benefits acquired by a pathogenic bacterium invading the host and causing an infection. A bacterial infection is the process occurring when the microbe manifests its pathogenicity, and thus its capacity of inducing disease, by invading and causing a damage (locally or systemically) of the host organism. Consequently, the infectious disease could result in an acute infection, with a short and severe course, or a chronic, low-grade and long lasting infection [26].

\section{Classification of bacterial pathogens}

The classification of infectious agents inregards to their infective lifestyles in the host and corresponding pathogenic indications must be precisely described [27]. In the life of a microbe, the intracellularity and extracellularity are unclear designations unless obviously related to the situation where it is living. For a microbial pathogen, what matters is whether intra-or extracellularity is in the basis of the in vivo life and in relationship with pathogenicity. Classically, infectious agents are indicated as extracellular and intracellular pathogens [28]

\section{Extracellular pathogens}

Staphylococcus aureus, Streptococcus pyogenes, Pseudomonas aeruginosa, Escherichia coli are typical examples of bacteria which have been considered extracellular pathogens, and lesion infections, osteomyelitis, scarlet fever, specified forms of pneumonia, urinary tract infections are examples of infections caused by these pathogens [29]. To produce disease, extracellular pathogens utilize any portal of entry provided a satisfactory fluid medium be recognized at the site of lesion [30]. Extracellular pathogens utilize virulence mechanisms to avoid the antimicrobial capabilities of humoral immunity and phagocytosis thus advancing extracellular reproduction, in contrast with intracellular pathogens that promote the entry in to host cells containing macrophages and non-professional phagocytes such as epithelial cells [31].

\section{Intracellular pathogens}

Classical examples of intracellular pathogens are Brucella abortus, Listeria monocytogenes, Mycobacterium tuberculosis, Salmonella enterica, and typical infectious diseases caused by theminclude brucellosis, listeriosis, tuberculosis, and salmonellosis [32]. Intracellular pathogenic bacteria have the ability to establish a relationship in the sensitive host which includes a stage of intracellular reproduction [33]. To establish an infection, these pathogens have to make contact with the appropriated type of host cell that provides suitable intracellular conditions for growth [34]. Bacteria such as Mycobacterium, Legionella, Brucella or Listeria have extended the ability to resist and replicate inside various mammalian cells including the aggressive phagocytic cells, which establish the first-line defense against invading pathogens [35].

\section{Targeted therapy of infections using nanoparticles}

The hydrophilic nature of some antibiotics prevents their capacity to penetrate the cells and, furthermore, the internalized molecules are mostly accumulated in lysosomes, where the bioactivity of the drug is low. Therefore, limited intracellular activity against sensitive bacteria is often found [36]. Thus, the use of drug delivery systems (DDS) has been suggested for passive targeting of infected cells of the mononuclear phagocytic system to enhance the therapeutic index of antimicrobials in the intracellular environment, while minimizing the side effects associated with the systemic administration of the antibiotic [37]. The pathophysiological and anatomical changes of the affected tissues in a disease state offer many possibilities for the delivery of various nanotechnology-based products [38]. Bacteria gains antibiotic resistance due to three reasons namely: 1) modification of active site of the target resulting in reduction in the efficiency of binding of the drug, 2) direct destruction or modification of the antibiotic by enzymes produced by the organism or, 3) efflux of antibiotic from the cell [39]. Nanoparticles (NPs) can target antimicrobial agents to the site of infection, so that higher doses of drug can be given at the infected site, thereby overcoming existing resistance mechanisms with fewer harmful effects upon the patient. As with nanoparticles targeting intracellular bacteria, nanoparticles targeting the site of infection can release high concentrations of antimicrobial drugs at the site of infection, while keeping the total dose of drug administered low. Nanoparticles can be targeted to sites of infection passively or actively. Passively targeted nanoparticles selectively undergo extravasation at sites of infection, where inflammation has led to enhanced blood vessel porousness. Actively targeted nanoparticles contain ligands (e.g. antibodies) that bind receptors (e.g. antigens) at sites of infection [40]. Passive targeting with nanoparticles, however, faces multiple barriers on the way to their target; these include mucosal barriers, nonspecific uptake of the particle and non-specific delivery of the drug (as a result of uncontrolled release) [41]. Passive nanoparticulate targeting of chemotherapeutics to the cells and organs of the reticuloendothelial system (RES) has been a significant area of research for the treatment of chronic infectious diseases. The RES comprises monocyte-lineage immune cells such as macrophages and dendritic cells, as well as the spleen, liver, and kidneys. These components of the RES are consistently implicated as sites of nanoparticle clearance and localization [42]. The few studies that have compared targeted and nontargeted systems have demonstrated that the role of targeting ligands in localization at the target site is application dependent. Targeted delivery to atherosclerotic lesions is greatly enhanced by targeting ligands which impart an improved ability to accumulate at the target site [43]. Many active targeting strategies use the enhanced permeability and retention (EPR) effect, so that active and passive targeting mechanisms act synergistically that lead to higher concentration of nanostructures in the infected region than that in healthy tissues [44]. Targeted antimicrobial drug delivery to the site of infection, particularly intracellular infections, using NPs is a sensational prevision in treating infectious diseases [45]. Intracellular microorganisms are taken up by alveolar macrophages (AMs), intracellulary survive or reproduce, and are persistent to the antimicrobial agents. Antibiotics loaded NPs can enter host cells through endocytosis, followed by releasing the payloads to delete intracellular microbes [46]. The need to target drugs to specific sites is increasing day by day as a result of therapeutic and economic factors. Nanoparticulate systems have shown enormous potential in targeted drug delivery, especially to the brain [24]

\section{Challenges in treating infectious diseases using nanotechnology}

Use of antibiotics began with commercial production of penicillin in the late 1940s and claimed to be a great success until the 1970-1980s when newer and even stronger antibiotics were additionally improved [47]. Resistance to antimicrobial drugs becomes a threatening problem not only in hospitals but also in communities, resulting in fewer effective drugs available to control infections by "old" well-known bacteria [48]. Carrier systems allow antibiotics to be delivered selectively to 
phagocytic cells and to increase their cellular penetration in order to treat intracellular infections, particularly in the case of antibiotics active against microorganisms that produce this type of infection but that have a low intracellular penetration capacity [49]. Nevertheless, significant challenges remain for implementation of clinically viable therapies in this field. New challenges in the development of nanotechnology-based drug delivery systems include: the possibility of scale-up processes that bring innovative therapeutic techniques to the market rapidly, and the possibility of obtaining multifunctional systems to carry out several biological and therapeutic requirements. Thus, a drug delivery system should be multifunctional and possess the ability to switch on and switch off specified functions when urgent. Another important requirement is that different properties of the multifunctional drug delivery systems are harmonized in an optimal fashion. Therefore, design, discovery, and delivery of antimicrobial drugs with improved efficacy and avoidance of resistance are extremely requested [50].

\section{Advantages of nanoantibiotics}

The use of NPs as delivery vehicles for antimicrobial agents suggests a new and promising model in the design of effective therapeutics against many pathogenic bacteria [51]. Antimicrobial NPs propose several clinical advantages. First, the surface properties of nanoparticles can be changed for targeted drug delivery for e.g. small molecules, proteins, peptides, and nucleic acids loaded nanoparticles are not known by immune system and efficiently targeted to special tissue types. Second, nanocarriers may overcome solubility or stability issues of the drug and minimize drug-induced side effects. Third, using nanotechnology, it may be possible to achieve co-delivery of two or more drugs or therapeutic modality for combination therapy. Fourth, NP-based antimicrobial drug delivery is promising in overcoming resistance to common antibiotics developed by many pathogenic bacteria. Five, administration of antimicrobial agents using NPs can progress therapeutic index, extend drug circulation (i.e., extended half-life), and achieve controlled drug release, increasing the overall pharmacokinetics. Six, the system can be used for several routes of administration including oral, nasal, parenteral, intraocular etc. Thus, antimicrobial NPs are of great interest as they provide a number of benefits over free antimicrobial agents [52].

\section{Nanotechnology-based drug delivery systems}

Perfectly, nanoparticulate drug delivery system should selectively accumulate in the necessary organ or tissue and at the same time, penetrate target cells to deliver the bioactive agent [53]. It has been proposed that, organ or tissue accumulation could be achieved by the passive or antibody-mediated active targeting, while the intracellular delivery could be mediated by specified ligands or by cell-penetrating peptides. The purpose of drug delivery is to carry out sustained (or slow) and/or controlled drug release and therefore to improve efficacy, safety, and/or patient comfort. Thus, the use of drug delivery systems has been suggested for passive targeting of infected cells of the mononuclear phagocytic system to enhance the therapeutic index of antimicrobials in the intracellular environment, while minimizing the side effects related with the systemic administration of the antibiotic. These systems propose many advantages in drug delivery, mainly focusing on improved safety and efficacy of the drugs, e.g. providing targeted delivery of drugs, improving bioavailability, extending drug or gene effect in target tissue, and improving the stability of therapeutic agents against chemical/ enzymatic degradation. The nanoscale size of these delivery systems is the basis for all these advantages. It is therefore assumed that, DDS with enhanced targeting property is highly promising in increasing the efficiency and efficacy of therapy while at the same time minimizing side effects.

\section{Types of drug carriers in medicine}

Polymeric nanoparticles: Polymer-based nanoparticles are submicron-sized polymeric colloidal particles in which a therapeutic agent of interest can be embedded or encapsulated within their polymeric matrix or adsorbed or conjugated onto the surface [54]. The drugs may also be sensitive to gastrointestinal degradation by digestive enzymes. The advantage of using polymeric nanoparticles is to permit encapsulation of bioactive molecules and protect them against enzymatic and hydrolytic degradation. Therapeutically used polymeric nanoparticles are composed of biodegradable or biocompatible materials, such as poly ( $\varepsilon$-caprolactone) (PCL), poly(lactic acid) (PLA), poly(lactic-co-glycolic acid) (PLGA), alginic acid, gelatin and chitosan. Polymeric nanocarriers (NCs) may suggest an opportunity to target chlamydial organism within the contents, as NCs have been shown to be excellent intracellular carriers, and can be appropriate to encapsulate a variety of therapeutics containing biomacromolecules. Compared to free drugs, polymeric NCs have many other advantages including improved drug bioa- vailability, high carrier capacity, the ability to release the payload in a controlled behavior and to adapt to different routes of administration and to concentrate in inflammatory and infectious locations by virtue of their enhanced permeability and preservation. Conjugating NCs with specific moieties have also been shown to enhance their targeting to specific cells and tissues. Polymeric nanoparticles have been extensively explored as means for drug solubilization, stabilization and targeting. Polymeric nanoparticles possess several unique characteristics for antimicrobial drug delivery. Firstly, polymeric nanoparticles are structurally stable and can be synthesized with a sharper size distribution. Secondly, particle properties such as size, zeta potentials, and drug release profiles can be accurately tuned by selecting different polymer lengths, surfactants, and organic solvents during the synthesis. Thirdly, the surface of polymeric nanoparticles typically contains functional groups that can be chemically changed with either drug moieties or targeting ligands. For targeted antimicrobial delivery, polymeric nanoparticles have been repeatedly ornamented with lectin, which is a protein that binds to simple or complex carbohydrates present on most bacterial cell walls. For example, lectin-conjugated gliadin nanoparticles were studied for treating Helicobacter pylori related infection diseases. It has been found that lectin-conjugated nanoparticles bind specially to carbohydrate receptors on cell walls of $H$. pylori and release antimicrobial agents into the bacteria. Rifampicin-loaded polybutylcyanoacrylate nanoparticles have also shown enhanced antibacterial activity both in vitro and in vivo against $S$. aureus and Mycobacterium avium due to an effective delivery of drugs to macrophages [45].

Hydrogels: A hydrogel is a network of hydrophilic polymers that can swell in water and hold a large amount of water while maintaining the structure [55]. Drugs can be loaded into the polymer matrix of these materials and controlled release is dependent on the diffusion coefficient of the drug across the hydrogel network. Amongst the several types of drug delivery systems that have been developed in order to improve effectiveness and biocompatibility, hydrogels are extremely promising. Hydrogels are biocompatible hydrophilic networks that can be constructed from both synthetic and natural materials. In an overall view, hydrogels can be classified based on a variety of characteristics, containing the nature of side groups (neutral or ionic), mechanical and structural features (affine or phantom), method of preparation (homoor co-polymer), physical structure (amorphous, semicrystalline, 
hydrogen bonded, supermolecular, and hydrocollodial), and responsiveness to physiologic environment stimuli $(\mathrm{pH}$, ionic strength, temperature, electromagnetic radiation, etc.). classically, hydrogels have been used to deliver hydrophilic, small-molecule drugs which have high solubilities in both the hydrophilic hydrogel matrix and the aqueous solvent swelling the hydrogel. Hydrogel-based hydrophobic drug delivery is in many respects a more difficult problem given the innate incongruity of the hydrophilic hydrogel network and the hydrophobic drug. A variety of strategies for introducing hydrophobic domains directly into otherwise hydrophilic hydrogel networks have permitted significant improvements in the loading of hydrophobic drugs. Hydrogel/glass composite (Nitric oxide-releasing nanoparticles) NO NPs have also been shown to have a high degree of effectiveness against (Methicillin-resistant Staphylococcusaureus) MRSA infection in several different mouse models. In one mouse study by Martinez et al., administration of topical hydrogel/glass composite NO NPs into skin wounds infected with MRSA reduced bacterial burden significantly compared to controls. Despite these many advantageous properties, hydrogels also have several limitations. The low elastic force of many hydrogels limits their use in load-bearing applications and can result in the precocious decomposition or flow away of the hydrogel from a targeted local site. This limitation may not be important in many typical drug delivery applications (e.g. subcutaneous injection) [56].

Metal nanoparticles: Metal-based nanoparticles of different shapes, sizes (between 10 to $100 \mathrm{~nm}$ ) have also been investigated as diagnostic and drug delivery systems. Most common metallic nanoparticles contain gold, nickel, silver, iron oxide, zinc oxide, gadolinium, and titanium dioxide particles Metal nanoparticles, which have a high specific surface area and a high fraction of surface atoms, have been studied extensively because of their unique physicochemical characteristics including catalytic activity, optical properties, electronic properties, antimicrobial activity, and magnetic properties. Even though metallic nanoparticles are biocompatible and immobile carriers, a significant fraction of metal particles can be retained and accumulated in the body after drug administration, probably causing toxicity. Consequently, the use of metallic nanoparticles for drug delivery is a concern [57].

Gold nanoparticles: Gold nanoparticles (GNPs) have found many applications in many fields such as cancer diagnosis and therapy, drug and gene delivery, DNA and protein determination, etc. Due to their unique properties of small size, large surface area to volume ratio, high reactivity to the living cells, stability over high temperatures and translocation into the cells [58]. GNPs are suitable for the delivery of drugs to cellular destinations due to their ease of synthesis, functionalization and biocompatibility. GNPs functionalized with targeted specific biomolecules can effectively destroy cancer cells or bacteria. The efficacy of GNPs conjugated to several antibiotics has also been the subject of some studies by Grace and Saha et al. They discovered that GNPs conjugates were more efficient in inhibiting the growth of Gram-positive and Gram-negative bacteria in comparison with the same dosage of antibiotics utilized alone. Their results suggest that GNPs can act as an effective drug carrier in a drug delivery system. Conjugates of gold nanoparticles with antibiotics and antibodies also have been used for selective photothermal killing of protozoa and bacteria. $\mathrm{Gu}$ et al. synthesized stable gold nanoparticles covered with vancomycin and showed significant enhancement of anti- bacterial activity, in comparison with the activity of the free antibiotic. In another report, Selvaraj et al. utilized the anticancer compound 5-fluorouracil bound to GNPs and found that the resulting conjugate was significantly more effective against a range of bacterial and fungal organisms in comparison with alone. Recently, it has been reported that the gentamicin conjugated with gold nanospheres was significantly more effective against $S$. aureus in comparsion with free gentamicin. Each GNP surrounded by a number of drug moieties acts as a single group against the microbial organisms. The greater antibacterial effect of the GNPs conjugates has been ascribed to their ability to bind to and/or penetrate the cell wall and, in doing so they are able to deliver a large number of antibiotic molecules into a highly localized volume.

Silver nanoparticles: Silver nanoparticles of size smaller than 100 $\mathrm{nm}$ contain about 10000-15000 silver atoms [59]. They are prepared by engineering the metallic silver into ultrafine particles by numerous physical methods, which include spark discharging, electrochemical reduction, solution irradiation and cryochemical synthesis. The most widely used and known application of silver nanoparticles is in the medical sciences. These include topical ointments and creams containing silver to prevent infection of burns and open wounds. Among the many different types of metallic and metal oxide NPs, silver nanoparticles have demonstrated to be the most effective against bacteria, viruses, and other eukaryotic microorganisms. Antibacterial properties inhibit the reproduction of bacteria, which is a microbe. The silver nanoparticles can "inactivate proteins, blocking respiration and electron transfer, and subsequently inactivating the bacteria". The antibacterial properties of the silver nanoparticles depend on the size of the particles; the smaller the particles the better the effect. The particle size is a major factor because the smaller the particle the greater the surface area, which allows for greater interaction with the bacteria. It has been reported that combined use of silver nanoparticles with antibiotics, such as penicillin G, amoxicillin, erythromycin, and vancomycin, resulted in enhanced and synergistic antimicrobial effects against Gram-positive and Gram-negative bacteria (e.g., E. coli and S. aureus). Although beneficial as antimicrobial agents, silver nanoparticles have adverse effects on cells such as the production of reactive oxygen species which are toxic to both bacteria and eukaryotic cells. In contrast, the cytotoxicity of gold nanoparticles is quite low, and they have been used for medical imaging and have served as scaffolds for drug delivery [60].

Magnetic nanoparticles: Magnetic nanoparticles engineered as drug delivery devices retain the ability to track their movement through the body. This is significant because it allows clinicians to monitor the effectivity of injected therapeutics to reach their target sites. Iron oxide nanoparticles (IONPs) are magnetic $\mathrm{Fe}_{3} \mathrm{O}_{4}$ or $\mathrm{Fe}_{2} \mathrm{O}_{3}$ nanocrystals which can interact with external magnetic fields, offering different opportunities in nanomedicine, e.g., as contrast agents in MRI, for magnetic hyperthermal therapies, or as magnetically triggerable drug delivery systems. There are some studies on evaluating the toxicity of magnetite nanoparticles on eukaryote cells, which their results showed negligible toxicity in eukaryote cells of the modified magnetite nanoparticles with different surfactants such as glycine or oleic acid. But the toxicity of magnetite nanoparticles on bacteria cells has not been reported. However, in most of the cases where magnetic nanocarriers have been used, difficulties in achieving these objectives appeared. In turn, magnetic force may not be strong enough to overcome the force of blood flow and to accumulate magnetic drugs only at target site. Therefore, designing magnetic drug delivery systems requires taking into consideration many factors, e.g., magnetic properties and size of particles, strength of magnetic field, drug loading capacity, the place of accessibility of target tissue, or the rate of blood flow. The vancomycin functionalized magnetic nanoparticles for pathogen detection have been investigated by $\mathrm{Gu}$ et al. Vancomycin 
can be attached to the magnetic nanoparticles surface by activating the $-\mathrm{COOH}$ group of vancomycin followed by reaction with the amine groups on the surface of the iron oxide nanoparticles. The vancomycin conjugated iron oxide nanoparticles were utilized as probes to selectively entrap S. saprophyticus (a pathogen that usually infects the urinary tract of young women) and $S$. aureus bacteria from urine specimen using a magnetic field. It has been reported that the various nanoparticles, $\mathrm{Al}_{2} \mathrm{O}_{3}, \mathrm{Fe}_{3} \mathrm{O}_{4}, \mathrm{CeO}_{2}, \mathrm{ZrO}_{2}$ and $\mathrm{MgO}$ were subjected to evaluate its antibacterial potential against ophthalmic pathogens such as Pseudo-monas aeruginosa, Acinetobacter sp., Klebsiella pneumoniae, E. coli, Streptococcus viridans andStreptococcus pyogenes. Among the nanoparticles, $\mathrm{Fe}_{3} \mathrm{O}_{4}$ showed maximum activity against Pseudomonas aeruginosa. The reactive oxygen species (ROS) generated by $\mathrm{Fe}_{3} \mathrm{O}_{4}$ nanoparticles could kill bacteria without harming nonbacterial cells [33].

Silica nanoparticles: Silica materials are suitable for several important biological applications, such as drug delivery, imaging, oxygen carrier or controlled release [61]. Silica materials have been proved to be efficient carriers for the local release of antibiotics, which could be of interest in the context of biofilm associated infections, which are a real challenge for the modern medicine. Moreover, mesoporous silica has been found to be relatively "non-toxic" and biocompatible, however of course depending on dose and administration route. Nanoporous silica materials possess large pore volumes and high surface areas, allowing the absorption of large amounts of drugs, thus providing sufficient concentrations for local treatment. The surface of silica materials is reactive due to the presence of silanol groups. This allows for facile modification by silanization reactions and thus opens possibilities for enhancing the drug loading and for controlling the drug release. Till present there are only few reports concerning the application of silica materials, crystalline or amorphous, in the antimicrobial therapy. Zhang et al. suggested a highly-sensitive fluoro immunoassay for the determination of staphylococcal enterotoxin $\mathrm{C}_{1}$

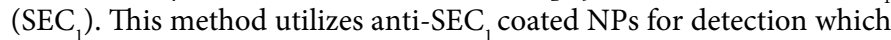
is possible in food samples and enables fluorescence microscopy imaging for the determination of SEC. Recently, Grumezescu et al. reported that silica nanostructures have significantly improved the anti-staphylococcal activity of bacitracin and kanamycin sulfate, as revealed by the drastic decrease of the minimal inhibitory activity of the respective antibiotics loaded in the $\mathrm{SiO}_{2}$ nanopowder. These results, correlated with the high biocompatibility of the porous silica structure recommend it as an efficient vehicle for the local delivery of antibiotics in lower active doses, reducing thus their cytotoxicity and side effects.

Micelles: Micelles are submicroscopic aggregates of surfactant molecules assembly of amphiphillic block copolymers or polymerlipid conjugates or other surface-active molecules that self-assemble in aqueous media to form structures with a hydrophobic core [62]. The ability to functionalize the micelles as well as tailor the disintegration behaviour by varying the co-polymer composition are beneficial parameters in making them drug carriers of choice. Their small size $(1-50 \mathrm{~nm})$ makes them ideal for intravenous delivery. In addition they are also more stable, when compared to liposomes due to be ability to design them to be chemically stable and biocompatible. One specific feature of micelles is that the amount of drug released can be controlled by an external stimulus like $\mathrm{pH}$, temperature, ultrasound or certain enzymes. Other unique properties of polymeric micelles are that they are easily altered with small functional groups that enhance their targeting potential. Generally, polymeric surfactants are known to be less toxic than low-molecular-weight surfactants, such as sodium dodecyl sulfate. Furthermore, in theory, polymeric micelles are considered very safe in relation to chronic toxicity. The disadvantage for the polymeric micelle systems is the immature technology for drug incorporation in a physical manner. Another disadvantage is much slower extravazation of polymeric carrier systems than that of low molecular weight drugs. This results from a difference in extravazation mechanisms between polymeric carrier systems and low molecular weight drugs.

Liposomes: Liposomes are small spherical vesicles in which one or more aqueous parts are completely surrounded by molecules that have hydrophilic and hydrophobic functionality. Liposomes change with composition, size, surface charge and method of preparation. They can be single or in multiple bilayers. Those including one bilayer membrane are called small unilamellar vesicles or large unilamellar vesicles based on their sizes [63]. Nanoparticulate DDS, such as liposomes, are mostly used to enhance the efficacy of drug and DNA delivery and targeting. Liposomes are also the most broadly used antimicrobial drug delivery vehicles because their lipid bilayer structure imitators the cell membrane and can readily fuse with infectious microbes. One of the disadvantages of liposomal antibiotics is the short shelf-lives of lipid vesicles, which limits drug stability. Short shelf lives can be conditioned by both physical and chemical processes. There are many advantages of liposomes as antibiotic carriers: improved pharmacokinetics and biodistribution; decreased toxicity; enhanced activity against intracellular pathogens; target selectivity; enhanced activity against extracellular pathogens, in particular to overcome bacterial drug resistance. The ability of liposomes to alter drug distribution depends mostly on their size and surface properties. Thus, liposomal encapsulation of antibiotics helps to increase their therapeutic index with mode of action related to increasing the drug concentration at the site of infection and/or reducing its toxicity. For instance, encapsulation of vancomycin and teicoplanin in liposomes resulted in significantly improved elimination of intracellular methicillin resistant $S$. aureus (MRSA) infection. Netilmicin liposomes showed an increase in pharmacologicalactivity in a peritonitis model of mice infected with $E$. coli, in terms of survival both prophylactically and therapeutically. Recently, Deol and Khuller produced lung-specific liposomes made of phosphatidylcholine, cholesterol, dicetylphosphate, O-steroyl amylopectin and mono sialo gangliosides/distearyl phosphatidyl ethanolamine-poly (ethylene glycol) 2000 for the targeted delivery of anti-Tuberculosis (TB) drugs to the lung [64].

Solid lipid nanoparticles (SLN): Solid lipid nanoparticles (SLN) were developed at the beginning of 1990s as an alternative carrier system to emulsions, liposomes and polymeric nanoparticles as a colloidal carrier system for controlled drug delivery. SLNs are sub-micron colloidal carriers, ranging from $50 \mathrm{~nm}$ to $1 \mu \mathrm{m}$, that are composed of physiological lipid dispersed in water or in aqueous surfactant solution [65]. In the last decade SLNs have gained considerable interest as novel particulate drug delivery systems. SLNs are suitable for the incorporation of lipophilic and hydrophilic drugs within the lipid matrix in considerable amounts. SLN consist of a solid lipid matrix at room and body temperature, where the drug is normally incorporated in the submicron size range (below $1 \mu \mathrm{m}$ ). Some advantages of SLNs are possibility of controlling drug release and drug targeting, increased drug stability, high drug payload, possibility of the incorporation of lipophilic and hydrophilic drugs, lack of biotoxicity of the carrier, no problems with respect to large-scale production, sterilization possibility, and good tolerability. Common disadvantages of SLN are their particle growing, their unpredictable gelation tendency, their unexpected dynamics of polymorphic transitions and their inherent low incorporation rate due to the crystalline structure of the solid lipid. SLNs are considered good drug carriers to obtain sustained 
release of antibiotics. SLNs can act as promising carriers for sustained ciprofloxacin release in infections or to enhance the bioavailability of tobramycin from antibiotic-loaded SLN in the aqueous humor for topical ocular delivery. Nimje et al. reported the selective delivery of rifabutin, another antituberculosis drug, to alveolar tissues, using drugloaded solid lipid nanoparticles, increasing the therapeutic margin of safety and reducing side effects. Another prominent example of SLNs-based drug delivery is pulmonary delivery of antimicrobials to treat tuberculosis, a serious lung infection caused by Mycobacterium tuberculosis. In some severe cases, tuberculosis infection spreads from the lungs and affects the lymphatic systems. SLNs can facilitate the delivery of anti-tuberculosis drugs such as rifampin, isoniazid and pyrazinamide to the lungs as well as to the lymphatic systems. Even though the development history of SLN-based antimicrobial drug delivery systems is relatively shorter than other nanoparticle systems such as liposomes and polymeric nanoparticles, SLNs have shown great therapeutic potentials.

Fullerenes: Fullerenes are a new form of carbon, other forms being diamond, graphite, and coal. They can take three forms of a hollow sphere, ellipsoid, or tube. Their small size, spherical shape, and hollow interior all provide therapeutic opportunities. The most abundant form of fullerenes is buckminsterfullerene (C60) with 60 carbon atoms arranged in a spherical structure. The shape of the molecule, recognized as truncated icosahedron, resembles that of a football ball, containing 12 pentagons and 20 hexagons, in which every carbon atom forms bond to three other neighbor atoms through $\mathrm{sp}^{2}$ hybridization. Friedman et al and Schinazi et al distinguished that the hydrophobic cleft of the human immunodeficiency virus (HIV)-1 protease can seamlessly host a C60 molecule. This discovery was the first piece of evidence that fullerenes could have pharmaceutical significance through interactions with biological targets, highlighting the great potential of fullerenes in medicinal applications. Since fullerenes possess unique geometrical shapes, as well as novel photophysical properties, in addition to being efficient radical scavengers, a wide variety of biological applications have been considered. Some studies asserted that C60 could be also utilized for the photodynamic inactivation of bacteria, as persuasively demonstrated in studies examining the effects of water-soluble and nanoparticulate $\mathrm{C} 60$ on various bacterial strains [66]. The effects were significantly more pronounced in Gram positive (Staphylococcus spp., Streptococcus spp.) than in Gram negative bacteria (Klebsiella pneumoniae, Escherichia coli, Pseudomonas aeruginosa, Salmonella typhi, Streptococcus pyogenes), indicating that the bactericidal action was dependenton the fullerene insertion into the microbial cellwall, the structure of which differs between Gram positive and Gram negative bacteria. Additionally, the quinazolin-fullerene conjugate 18 was reported to have an inhibitory potential of $98.83 \%$ at a minimal inhibitory concentration of $1.562 \mu \mathrm{g} / \mathrm{mL}$ when treating $M$. tuberculosis.

Dendrimers: First discovered in the early 1980's by Tomalia and coworkers, such hyperbranched molecules were called dendrimers [67]. Dendrimers are globular repeatedly branched macromolecules that exhibit controlled patterns of branching with multiple arms extending from a central core. The well-defined structure, monodispersity of size, surface functionalization capability, and stability are properties of dendrimers that make them attractive drug carrier candidates. Asymmetric dendrimers are synthesized by coupling dendrons of different generations (G1-G4) to a linear core, which yields a branched dendrimer with a non-uniform orthogonal architecture. This asymmetry allows for tunable structures and molecular weights, with precise control over the number of functional groups available on each dendron for attachment of drugs, imaging agents, and other therapeutic moieties. Dendrimers also possess many unique properties that make them a good nanoparticle platform for antimicrobial drug delivery. They are highly arranged and regularly branched globular macromolecules, with a core, layers of branched repeat units emerging from the core and functional end groups on the outer layer of repeat units. Dendrimer biocides may contain quaternary ammonium salts as functional end groups displaying greater antimicrobial activity against bacteria than small drug molecules, due to a high density of active antimicrobials on the dendrimer surfaces. Dendrimers can be made from a wide variety of biocompatible materials; the most frequently used are polyamidoamine (PAMAM), polyethylene oxide (PEO), polypropylene imine (PPI), polyethyleneimine (PEI), polyethylene glycol (PEG) etc. PAMAM dendrimers are dendritic polymers characterized by regular branching and radial symmetry. PAMAM dendrimers have illustrated useful drug delivery and antimicrobial applications with amino-terminated dendrimers showing high antibacterial efficacy. It is well known that PAMAM dendrimers with primary amine surface functional groups may enter the cellular membrane. Sulfomethoxazole (a sulfonamide derivative poorly soluble and thus presenting low bioavailability) was administered with PAMAM dendrimers in vitro. Sulfamethox-azole (SMZ)-encapsulating PAMAM dendrimers led to sustained release of the drug in vitro and 4-8 folds increased antibacterial activity against $E$. coli, compared to free SMZ.

Zeolites: Zeolites are solid hydrated crystalline materials with frame-works comprising silicon, aluminum and oxygen and featuring nano-channels and cages of regular dimensions. Silica is a neutral regular tetrahedronin in which positive charge of silicon ion is balanced by oxygen [68]. The capacity of cation exchange depends on the ratio of silica/alumina in the structure. Generally, zeolits with a low silica/alumina ( $\mathrm{Si} / \mathrm{Al})$ ratio have higher ion exchange capacity. According Si/Al ratio, there are several types of natural and synthetic zeolites including zeolite- $\beta$, zeolite $\mathrm{A}$, zeolite $\mathrm{X}$ and zeolite $\mathrm{Y}$, which are the most common commercial adsorbents. Zeolites are minerals with selective pores that can be used to sieve molecules having certain dimensions. Several recent studies showed that the potential of zeolites in medical applications is due to their structural properties and stability in biological environments. Zeolites have also been explored as suitable hosts for the encapsulation of drug molecules, in search for efficient drug delivery systems. Both zeolites and drugs have been administrated simultaneously to a patient without loss of the individual pharmacological effect of the drugs. Coating or impregnating zeolite with metallic silver nanoparticles to prepare zeolite composites can enhance the antibacterial ability of materials, and these materials can inhibit bacterial growth effectively. It has been reported that silver embedded zeolite A was found to be antibacterial against $E$. coli, Bacillus subtilis and staphy-lococcus aureus. Moreover, polymer composites of plasticized poly (vinylchloride) pellets with silver zeolites demonstrated activity against $S$. epidermidis and $E$. coli, while polyurethane composites with silver zeolites showed antimicrobial action against $E$. coli and polylactid acidpolylactide (PLA)/silver zeolite composites also presented activity against $S$. aureus and $E$. coli, with silver being effectively released from the films.

Quantum dots: Quantum dots (QDs) are nanocrystals formed by semiconductor materials, showing attractive photophysical properties, containing high quantum yield, resistance to photobleaching, and harmonic photoluminescence, making them potentially powerful tools in a range of biomed- ical applications. QDs are typically in the size range between $1 \mathrm{~nm}$ and $10 \mathrm{~nm}$, composed of groups II-VI (e.g., CdSe) or II-V (e.g., InP) elements of the periodic table. QDs are highly 
bright, photostable and possess high quantum yield. Due to their very small size, they possess unique properties and behave in different way than crystals in macro scale. Water-soluble QDs may be crosslinked to biomolecules such antibodies, oligonucleotides, or small molecule ligands to render them specific to biological targets. A variety of techniques have been explored to label cells internally with QDs, using passive uptake, receptor-mediated internalization, chemical transfection, and mechanical delivery. QDs have been loaded passively into cells by exploiting the innate capacity of many cell types to uptake their extracellular space through endocytosis. Krauss group utilized CdSe/ZnS streptavidin-coated QDs to detect solitary pathogenic E. coli O157:H7 in phosphate buffer saline solution. Biotinylated anti E. coli O157:H7 distinguished streptavidin-coated QDs via famous avidinbiotin binding. Once treated, QD labeled antibody selectively targeted pathogenic E. coli O157:H7 over common lab strain E. coli DH5a. This assay represented 2 orders of magnitude more sensitivity than using an organic dye with minimal non-specific binding between the QDs and the bacterial cells. Recently, Luo et al. reported that CDTe QDs coupled to a rocephin antibiotic complex exhibited antibacterial activity against Escheri-chia coli. The mechanism for the antimicrobial activity of QDs is unclear, but it is possiblethat QDs can produce singlet $\mathrm{O}_{2}$, a source of free radicals, under irradiation. Heavy metal ion oxides can also form the QDs core and result in antimicrobial activity. A recent and excellent review emphasized the application of bioconjugated quantum dots for the detection of food contaminants such as pathogenic bacterial toxins like botulinum toxin, enterotoxins produced by Staphylococcus aureus and Escherichia coli.

\section{Antibacterial activity of carrier systems for intracellular infection}

Treatment of intracellular bacterial infection remains both a medical and economic challenge. Pathogens thriving or maintaining themselves in cells, or simply taking transient refuge therein, are indeed shielded from many of the humoral and cellular means of defense. They also seem more or less protected against many antibiotics [69]. Various infectious diseases are caused by facultative organisms that are able to survive in phagocytic cells. The intracel- lular location of these microorganisms protects them from the host defence systems and from some antibiotics with poor penetration into phagocytic cells. Intracellular infections are especially difficult to eradicate because bacteria fight for their survival using several ingenious mechanisms: inhibition of the phagosome-lysosome fusion, resistance to attack by lysosomal enzymes, oxygenated compounds and defensins of the host macrophages, escape from the phagosome into the cytoplasm. Thus, the need for the development of improved antimicrobial chemotherapeutics and prophylaxis strategies is increasing. In spite of the availability of a wide variety of in vitro active antibiotics, therapeutic deficiencies are reported, mainly because of the inability of the drugs to reach the bacteria harboring intracellular compartments or to perform their activity in the intracellular environments. However, the poor cellular penetration limits this use in the treatment of infections caused by intracellular pathogens. One strategy utilized to improve the penetration of antibiotics into phagocytic cells is the use of carrier systems that deliver these drugs directly to the target cells. Several in vivo and in vitro studies have reported the potential applications of various carrier systems to enhance the selectivity of antibiotics for phagocytic cells and sustain therapeutic efficiency in the treatment of intracellular infections.

\section{Infections due to mycobacteria}

Tuberculosis, caused by Mycobacterium tuberculosis, is a ordinary lung infection that is even endemic to specified regions. Its prevalence has increased recently because it is often associated with AIDS. The Mycobacterium avium complex (MAC) complex is the main cause of hardships in immunodepressed patients. There are drugs that are efficient against tuberculosis, but these are used in extended treatment, increasing the risk of side effects. Moreover, tuberculosis has emerged as an occupational disease in the health care set-up. Although an effective therapeutic regimen is available, patient noncompliance (because of the need of taking antitubercular drugs daily or several times a week) results in treatment failure as well as the emergence of drug resistance. The use of delivery systems facilitates the selective shuttling of antibiotic to the site of infection and such systems provide slow and prolonged drug release, which permits administration over longer intervals of time. The encapsu- lation of antitubercular drugs in polymeric particles is another strategy to improve the current therapeutic regimen of tuberculosis. In the last few years several antitubercular drugs-containing PLGA and PLA microparticles and mainly nanoparticles have been comprehen- sively studied. Fawaz et al. encapsulated the synthetic drug ciprofloxacin in polyisobutylcyanoacrylate (PIBCA) nanoparticles. When testing these nanoparticles against a $M$. avium infection in a human macrophage culture, it was found that though nanoparticleassociated ciprofloxacin was more effective than unbound ciprofloxacin, it was much less so than anticipated. Rifampicin-loaded polybutylcyanoacrylate nanoparticles have shown enhanced antibacterial activity both in vitro and in vivo against S. aureus and M. avium due to an effective delivery of drugs to macrophages. The encapsulation of different antibiotics in liposomes has shown good antibacterial efficacy in both macrophage cell lines and in animal models of MAC-due disease [70]. Ciprofloxacin efficiently inhibits the growth of $M$. avium invitro in a murine macrophage-like cell line using negatively charged liposomes and in vivo using specific stealth liposomes in a mouse model of tuberculosis infection. Similar results have been obtained using stealth liposomes of isoniazid and rifampicin, which show controlled release and reduce toxicity in vivo in mice infected with $M$. tuberculosis.

\section{Brucellosis}

Brucellosis is an infectious disease caused by Brucella spp. Four species, Brucella abortus, Brucellamelitensis, Brucella suis and Brucella canis, have been recognized as human pathogens each associated with a different natural host animal [71]. These small coccobacilli are mainly localized intracellularly within phagocytic cells making treatment difficult, since most antibiotics, although highly active in vitro, do not actively pass through cellular membranes. However in the last two decades many experiments have provided good evidence criteria for its antibiotic treatment, the most suitable antimicrobial therapy for human brucellosis continues to be a controversial subject. Because of its intracellular location, long treatments with several antibiotics are required. Relapses are frequent owing to the low efficacy of many drugs and the lack of patient agreement. Thus, alternative methods such as drug delivery systems to achieve high intracellular bactericidal activity should be considered. Gentamicin, encapsulated in different types of liposomes, has been evaluated against murine monocytes infected with B. abortus. All such liposomes reduced the number of bacteria, the most effective being SPLVs (stable plurilamellar vesicles). Rifampicin-loaded mannosylated dendrimers have indicated specific $\mathrm{pH}$-dependent delivery of this antibiotic to rat alveolar macrophages. Recently, gentamicin loaded poly (D, L-lactide-co-glycolide) (PLGA) have been obtained by the several emulsion solvent evaporation method for the treatment of brucellosis. Thus, alternative methods such as DDS to achieve high intracellular bactericidal activity seem promising. The 
possible use of drug delivery systems containing aminoglycosides may be one of the most appropriate therapeutic advances in human brucellosis treatment in the recent years.

\section{Salmonellosis}

Salmonellosis is one of the most serious food-borne diseases affecting humans. It may be considered the most important pandemic zoonosis under natural conditions. Bacteria of the genus salmonella are facultative intracellular parasites that cause salmonellosis and typhoid fever. Antibiotics effective against this type of bacteria have limitations owing to the problems of formulation, low penetration, or the appearance of side effects; these can be solved using carrier systems. Several studies using antibiotic-loaded nanoparticles have been performed in order to recognize the suitability and efficacy of these carriers in experimental models of salmonellosis. In order to recognize whether polyalkycyanoacrylate nanoparticles were also effective against nondividing bacteria, Page-Clisson et al. studied the effectiveness of these carriers in a model of persistent Salmonella typhimurium infection. They found that although at early stages of the infection, when bacteria are actively dividing, there was an antibacterial effect, neither free nor nanoencapsulated ciprofloxacin or ampicillin could significantly reduce infection in the liver or the spleen at later stages. Liposomal ciprofloxacin, administered intravenously and intraperitoneally to mice infected with intracellular $S$. typhimurium, has increased habitation time in plasma and the concentration of drug in the liver, spleen, lungs and kidneys is also increased, while when administered intratracheally its pulmonary retention is increased. Compared with free ciprofloxacin, it extends survival and reduces the number of bacteria in the liver and spleen. Therefore, alternative methods such as DDS which achieve high protective and bactericidal activity should be taken into account in the future as suitable treatments for Salmonellainduced infections.

\section{Lysteriosis}

Lysteria monocytogenes is a facultative intracellular parasite able to cause meningitis andsepticaemia. The encapsulation of ampicillin in liposomes decreases the survival of L. mono-cytogenes in mouse peritoneal macrophages to different extents, depending on the compositionof the liposomes. Chitosan-coated plastic films, alone or loaded with antimicrobial agents, were evaluated for their effect against L. monocytogenes. These chitosan-coated films inhibited this pathogen growth in a concentration-dependent manner whereas chitosancoated films impregnated with antibiotics were significantly more effective against L. monocytogene. Formulation of gentamicin in liposomes containing DOPE (dioleylphosphatidylethanolamine) and sensitive to $\mathrm{pH}$ has been reported to increase the concentration of drug in mouse macrophages infected with $L$. monocytogenes, increasing its bactericidal activity. This formulation is more effective against $L$. monocytogenes than against other bacteria owing to its location in the cytosol. Furthermore, the efficacy of liposomes and free antibiotic were distinguished in Listeria-infected mice. Seven days after the treatment, ampicillin-loaded liposomes hadreduced the infection by 3.2 logs in the liver and 2.8 logs in the spleen, while free ampicillin was ineffective. In another example, ampicillin-encapsulated polyisohexylcyanoacrylate nanoparticles have been investigated against L. monocytogenes in mouse peritoneal macro- phages [71]. Synthetic biodegradable and biocompatible polymers have been shown to be effective for encapsulating a great variety of antibiotics. In addition, these polymeric particles powerfully enhance phagocytosis and are suitable for intracellular delivery of antibacterial agents. With the continuous attempts in this field, there is no doubt that nanoparticlebased drug delivery systems will continue to improve treatment to bacterial infections, particularly in life-threatening diseases such as tuberculosis infections.

\section{Development of Nanoparticles for Antimicrobial Drug Delivery}

Upon invasion of the epithelial surfaces, infectious microorganisms spread throughout the body via the circulatory system. They are then removed from the blood by macrophages which are present in all major organs such as liver, spleen and bone marrow. After being phagocytosed by macrophages, the infectious microorganisms are trapped in phagosomes, which then fuse with lysosomal granules inside cell cytoplasm forming phagolysosomes. Subsequently, oxygendependent or oxygen-independent bacterial killing mechanisms induced by enzymes inside the phagolysosomes occur to digest the trapped microorganisms. However, many microorganisms are able to evade the macrophage digestion via escaping from the phagosomes, inhibiting the phagosome-lysosome fusion, withstanding the lysosomal enzymes, or resisting oxidative and non-oxidative killing mechanisms. These bacterial defense mechanisms make intracellular infections difficult to eradicate resulting in infec-tious diseases that range from staph infections to tuberculosis. An antimicrobial refers to a substance that kills or inhibits the growth of microorganisms. Since the discovery of antimicrobial drugs in the 1960s, many infectious diseases have been overcome. Typically, antimicrobials kill bacteria by binding to some vital compounds of bacterial metabolism, thereby inhibiting the synthesis of functional biomolecules or impeding normal cellular activities. For instance, lactams such as penicillins and cephalosporins inhibit bacteria cell wall synthesis; tetracyclines, macrolides, and clindamycin inhibit protein synthesis; metronidazole and quinolones inhibit nucleic acid synthesis; and sulphonamides and trimethoprim have an inhibitory effect on enzyme synthesis. Some antimicrobials such as penicillin are only effective against a narrow range of bacteria, whereas others, like ampicillin, kill a broad spectrum of Gram-positive and Gram-negative bacteria. Despite the great progress in antimicrobial development, many infectious diseases, especially intracellular infections, remain difficult to treat. One major reason is that many antimicrobials are difficult to transport through cell membranes and have low activity in-side the cells, thereby imposing negligible inhibitory or bactericidal effects on the intracellular bacteria. In addition, antimicrobial toxicity to healthy tissues poses a significant limitation to their use. Aminoglycosides, for instance, cause ototoxicity and nephrotoxicity and have to be given in con-trolled dosages. Another major issue with antimicrobials stems from the acquired resistance of infectious microbes. In 2002, more than $70 \%$ of bacteria causing hospital-acquired infections were resistant to at least one common antimicrobial in the United States.

Over the last few decades, the applications of nanotechnology in medicine have been extensively explored in many medical areas, especially in drug delivery. Nanotechnology concerns the understanding and control of matters in the 1-100 nm range, at which scale materials have unique physicochemical properties including ultra small size, large surface to mass ratio, high reactivity and unique interactions with biological systems. By loading drugs into nanoparticles through physical encapsulation, adsorption, or chemical conjugation, the pharmacokinetics and therapeutic index of the drugs can be significantly improved in contrast to the free drug counterparts. Many advantages of nanoparticle-based drug delivery have been recognized, including improving serum solubility of the drugs, prolonging the systemic circulation lifetime, releasing drugs at 
a sustained and controlled manner, preferentially delivering drugs to the tissues and cells of interest, and concurrently delivering multiple therapeutic agents to the same cells for combination therapy. Moreover, drug-loaded nanoparticles can enter host cells through endocytosis and then release drug payloads to treat microbes-induced intracellular infections.

As a result, a number of nanoparticle-based drug delivery systems have been approved for clinical uses to treat a variety of diseases and many other therapeutic nanoparticle formulations are currently under various stages of clinical tests. Knowing the vast scope of nanoparticle drug delivery, here we will only focus on the development and application of nanoparticles for antimicrobial drug delivery through various mechanisms. As listed in Figure 1, a few types of nanoparticles including liposomes, polymeric nanoparticles, solid lipid nanoparticles and dendrimers have been widely investigated as antimicrobial drug delivery platforms, of which several products have been introduced into pharmaceutical market. This review will summarize the current status, mechanisms of action, and structure-activity relation-ship of these nanoparticle-based antimicrobial delivery systems.

\section{Discovery of Dendrimers and Dendritic Polymers: A Brief Historical Perspective}

One of the most pervasive topologies observed on our planet is perhaps dendritic architecture. Innumerable examples of these patterns may be found in both abiotic systems as well as in the biological world. In biological systems, these den-dritic patterns may be found at dimensional length scales measured in meters (trees), millimeters/ centimeters (fungi), or microns (neurons) as illustrated in Figure 1. The reasons for such extensive mimicry of these dendritic topologies at virtually all dimensional length scales is not entirely clear. However, one might speculate that these are evolutionary architectures that have been optimized over the past several billion years to provide structures manifesting maximum interfaces for optimum energy extraction/ distribution, nutrient extraction/distribution, and information storage/retrieval. The first inspiration for synthesizing such molecular level treelike structures evolved from a lifetime hobby enjoyed by one of the authors (D. A. Tomalia) as a horticulturist/tree grower. Although perhaps first con-ceptualized by Flory, the first successful laboratory synthesis of such dendritic complexity did not occur until the late 1970s. It required a significant digression from traditional polymerization strategies with realignment to new perspectives. These perspectives utilized major new synthesis concepts that have led to nearly monodispersed synthetic macromolecules. This was the first time in the history of synthetic polymer science that precise abiotic macromolecules could be synthesized without the use of a biological system. The result was a unique core-shell macromolecular architecture, now recognized as den-drimers. The concept of repetitive growth with branching was first reported in 1978 by Buhleier et al. (University of Bonn, Germany) who applied it to the construction of low molecular weight amines. This was followed closely by the parallel and independent development of the divergent, macromolecular synthesis of "true dendrimers" in the Tomalia group (Dow Chemical Co.). The firstarticle using the term "dendrimer" and describing in great detail the preparation of poly (amidoamine) (PAMAM) dendrimers was presented in 1984 at the 1st International Polymer Conference, Society of Polymer Science, Japan (SPSJ). It was then published in 1985, the same year a communication reported the synthesis of arborols by Newkome et al.

The divergent methodology based on acrylate mono-mers was discovered in 1979 and developed in the Dow laboratories during the period of 1979-985. It did not suffer from the problem of low yields, purity, or purifi-cation encountered by Voegtle in his "cascade" synthesis and afforded the first family of well-characterized dendrimers. PAMAM dendrimers with molecular weights ranging from several hundred to over 1 million daltons (i.e., generations 1-13) were prepared in high yields. This original methodology was so successful that today it still constitutes the preferred commercial route to the trademarked Starburst dendrimer family. It is both remarkable and surprising to find that many of these Class IV dendritic structurecontrolled macromolecules (i.e., dendrimers, dendrigrafts, etc.) possess

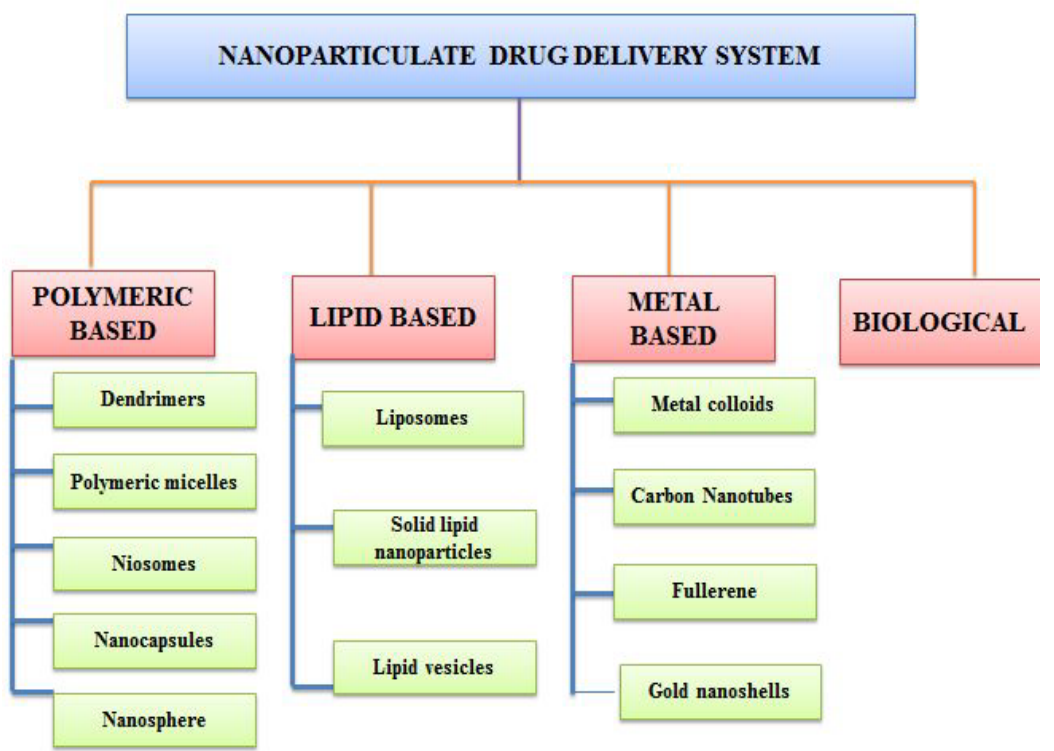

Figure 1: Different types of nano-devices for delivery of antibacterial agents 
topologies, function, and dimensions that scale very closely to a wide variety of important biological polymers and assemblies. Dendritic polymers, more specifically dendrimers, are expected to play a key role as an "enabling technology" in this challenge during the next century. Justas the first three traditional, synthetic polymer architectures have so successfully fulfilled the critical material and functional needs for society during the past half-century, it is appropriate to be optimistic about such a role for the "dendritic state".

\section{Dendrimers for Antimicrobial Drug Delivery}

Dendrimers are defined as highly ordered and regularly branched globular macromolecules produced by stepwise iterative approaches. The structure of dendrimers consists of three distinct architectural regions: a focal moiety or a core, layers of branched repeat units emerging from the core, and functional end groups on the outer layer of repeat units. In 1978, the first iterative cascade synthetic procedure for branched amines was discovered by Vögtle et al. A few years later, highly branched l-lysine-based dendrimers were patented. In 1984, Tomalia et al. reported the synthesis and characterization of the first family of polyamidoamine (PAMAM) dendrimers, which has become one of the most popular dendrimers since then.

Two synthetic approaches, divergent and convergent approaches, have been developed to synthesize dendritic systems for delivering various types of drugs. The divergent approach initiates the synthesis from a core and emanates outward through a repetition of coupling and activation steps. During the first coupling reaction, the peripheral functional groups of the core react with the complementary reactive groups to form new latent branch points at the coupling sites and increase the number of peripheral functional groups. These latent functional groups are then activated to couple with additional monomers. The activation of the la-tent functional groups can be achieved by removal of protecting groups, coupling with secondary molecules, or reactive functionalization. Large excess of reagents is required to drive the activation step to completion. The final resulted dendrimer products can be separated from the excess reagents by distillation, precipitation or ultrafiltration. Although the divergent approach is ideal for large-scale production, incomplete functionalization or side reactions can occur when the number of generation increases. These flawed dendrimers are usually difficult to be separated from the final products because of structural similarity. In contrast, the convergent approach initiates the synthesis from the periphery and progresses inward. This approach starts with coupling end groups to each branch of the monomer, followed by the activation of a single functional group located at the focal point of the first wedge-shape dendritic fragment or dendron. Higher generation dendron is synthesized by the coupling of the activated dendron to an additional monomer. After repetition of coupling and activation step, a globular dendrimer is formed by attaching a number of dendrons to a polyfunctional core. Dendrimers thus synthesized can be effectively purified. However, synthesis of large dendrimers above the sixth generation is difficult.

Dendrimers possess several unique properties that make them a good nanoparticle platform for antimicrobial drug delivery. The highly-branched nature of dendrimers provides enormous surface area to size ratio and allows great reactivity with microorganisms in vivo. In addition, both hydrophobic and hydrophilic agents can be loaded into dendrimers. Hydrophobic drugs can be loaded inside the cavity in the hydrophobic core, and hydrophilic drugs can be attached to the multivalent surfaces of dendrimers through covalent conjugation or electrostatic interaction. Moreover, by using antimicrobial drugs as a building block, the synthesized dendrimers themselves can become a potent antimicrobial. Dendrimer biocides are such example that contains qua-ternary ammonium salts as functional end groups. Quaternary ammonium compounds (QACs) are antimicrobial agents that disrupt bacterial membranes. Dendrimer biocides have displayed greater antimicrobial activity against target bacteria than small drug molecules because of a high density of active antimicrobials present on the dendrimer surfaces. The polycationic structure of dendrimer biocides facilitates the initial electrostatic adsorption to negatively charged bacteria. The absorption then increases membrane permeability and allows more dendrimers for entering the bacteria, leading to leakage of potassium ions and eventually complete disintegration of the bacterial membrane. PAMAM is one of the most studied dendrimers for an-timicrobial delivery because of its higher density of func-tional groups, which make the dendrimer more hydrophilic and more readily reactive to antimicrobial conjugation. Silver salts loaded PAMAM dendrimers have demonstrated significant antimicrobial activity against Staphylococcusaureus, Pseudomonas aeruginosa, and Escherichia coli. Incorporation of antibacterial agents such assulfamethoxa-zole (SMZ) into the ethylenediamine (EDA) core of PAMAM dendrimers has significantly improved the drug's aqueous solubility and antibacterial activity against $E$. coli. Many other antimicrobial drugs have been successfully loaded into dendrimer nanoparticles and have shown improved solubility and therapeutic efficacy. In spite of the great progresses on nanoparticlebased antimicrobial drug delivery, here we call attention to the need to unite the shared interest between nanoengineers and microbiologists in developing novel nanotechnology targeting a few major unmet challenges of antimicrobial drug delivery. First, acquired microbial drug resistance remains a major challenge for infection treatment. One possible approach is to incorporate more than one antimicrobial drug to a single nanoparticle and then concurrently deliver the drugs to the same microbes. Combinatorial drug therapy is expected to have higher potency as multiple drugs can achieve synergistic effects and overwhelm microbial defense mechanisms. Secondly, premature drug release from the antimicrobial-loaded nanoparticles remains another major challenge, especially for treating systemic and intracellular infections. To minimize drug loss before the nanoparticles arrive at the infectious sites, an infection microenvironment-sensitive drug release nanoparticles can be developed. That is, negligible amount of antimicrobial drugs will be released when the nanoparticles circulate in the blood stream or encounter healthy tissues whereas triggered rapid drug release will occur after the nanoparticles get to the infectious cells or tis-sues. Potential drug-release triggers include $\mathrm{pH}$ value, enzyme and other unique characters of the infection microenvironment. Lastly, few of the current nanoparticle-based antimicrobial drug delivery systems can distinguish microbes or infectious cells from healthy cells due to the lack of the specific targeting ability, although targeted drug delivery has been extensively studied for other disease treatment such as cancers and cardiovascular diseases. It would be beneficial for infection treatment if antimicrobial nanoparticles could be modified with microbe antigen- or infectious cell antigen-specific ligands including antibodies, antibody fragments, aptamers and peptides.

\section{Physicochemical Properties of Dendrimers}

As the dendrimer grows, the different compartments of the dendritic structure begin to show distinct features which are amplified with increasing generation. The dendrimer structure may be divided into three parts:

- A multivalent surface, with a high number of functionalities. 
Dependent on the dendrimer generation, the surface may act as a borderline shielding off the dendrimer interior from the surroundings. This increasingly "closed" surface structure may result in reduced diffusion of solvent molecules into the dendrimer interior.

- The outer shell, which have a well-defined microenvironment, to some extent shielded from the surroundings by the dendrimer surface. The very high number of functionalities located on the surface and the outer shell are well-suited for host-guest interactions and catalysis where the close proximity of the functional motifs is important.

- The core, which as the dendrimer generation increases, gets increasingly shielded off from the surroundings by the dendritic wedges. The interior of the dendrimer creates a microenvironment which may have very different proper-ties compared to the surroundings. For example as decribed elsewhere, water-soluble dendrimers with an apolar interior have been constructed to carry hydrophobic drugs in the bloodstream [43].

\section{Dendrimers and the effect of molecular growth}

The conformational behaviour of adendrimer upon growing to higher generations are determined by the molecular dimensions of the monomers-short monomers induce rapid proliferation of chains within a small space, the flexibility of the dendrons and the ability of the end-groups to interact with each other, e.g. by hydrogen bonding creating a dense outer shell. Dendrimers and the effect of $\mathrm{pH}$ : Aminoterminated PPI and PAMAM dendrimers have basic surface groups as well as a basic interior. For these types of dendrimers with interiors containing tertiary amines, the low $\mathrm{pH}$ region generally leads to extended conformations due to electrostatic repulsion between the positively charged ammonium groups.

\section{Dendrimers and the effect of solvent}

The ability of the solvent to solvate the dendrimer structure is a very important parameter when investigating the conformational state of a dendrimer. Molecular dynamics has been applied to study the variation of dendrimer conformation as a function of dendrimer generation in different solvents. Dendrimers of all generations generally all experience a larger extend of back-folding with decreasing solvent quality, i.e. decreasing solvation. However, being more flexible, the low generation dendrimers show the highest tendency towards backfolding as a result of poor solvation compared to the higher generation dendrimers.

\section{Dendrimers and the effect of salt}

Molecular simulations generally conclude that high ionic strength (high concentration of salts) has a strong effect on charged PPI dendrimers and favours a contracted conformation of dendrimers, with a high degree of back-folding somewhat similar to what is observed upon increasing $\mathrm{pH}$ or poor solvation. At low salt conditions, the repulsive forces between the charged den-drimer segments results in an extended conformation in order to minimise charge repulsion in the structure.

\section{Dendrimers and the effect of concentration}

In dendrimers with flexible structures the conformation is not only affected by small molecules like solvents, salts or protons, but may also be sensitive to larger objects, such as other dendrimers or surfaces which can have a great affect on the molecular density and conformation of the dendrimer. Small angle X-ray scattering (SAXS) experiments performed on PPI dendrimers $(\mathrm{G} 4, \mathrm{G} 5)$ in a polar solvent like methanol show that the molecular conformation of dendrimers upon increasing concentration becomes increasingly contracted. This molecular contraction may minimise the repulsive forces between the dendrimer molecules and increase the ability of the dendrimers to exhibit a more tight intermolecular packing.

\section{Type of Dendrimers}

\section{PAMAM dendrimer}

Poly (amidoamine) dendrimers (PAMAM) are synthesized by the divergent starting ammonia method from a ethylene diamine initiator core. PAMAM dendrimers are commercially available, usually as methanol solutions. Starburst dendrimers is applied as a trademark name for a sub-class of PAMAM dendrimers based on a tris-aminoethylene-imine core. The name refers to the star like pattern observed when looking at the structure of the high-generation dendrimers of this type in two-dimensions.

\section{PAMAMOS dendrimer}

Radially layered poly (amidoamine-organosilicon) dendrimers (PAMAMOS) are inverted unimolecular micelles that consist of hydrophilic, nucleophilic polyamidoamine (PAMAM) interiors and hydrophobic organosilicon (OS) exteriors. These dendrimers are exceptionally useful precursors for the preparation of honeycomblike networks with nanoscopic PAMAM and OS domains. These are silicone containing first commercial dendrimers.

\section{PPI dendrimer}

PPI-dendrimers stand for "Poly (Propylene Imine)" describing the propylamine spacer moieties in the oldest known dendrimer type developed initially by Vogtle. These dendrimers are generally polyalkyl amine, having primary amines as end groups; the dendrimer interior consists of numerous of tertiary tris-propylene amines. PPI dendrimers are commercially available up to G5, and has found widespread applications in the field of material science and biology. As an alternative name to PPI, POPAM is sometimes used to describe this class of dendrimers. POPAM stands for Poly (Propylene Amine), which closely resembles PPI.

\section{Tecto-dendrimer}

These are composed of a core dendrimer, surrounded by dendrimers of several steps to perform a function necessary for a smart therapeutic nanodevice. Different compounds perform varied functions ranging from diseased cell recognition, diagnosis of disease state, drug delivery, reporting outcomes of therapy.

\section{Multilingual dendrimers}

In these dendrimers, the surface contains multiple copies of a particular functional group.

\section{Chiral dendrimers}

The chirality in these dendrimers is based upon the construction of a constitutionally different but chemically similar branch to chiral core.

\section{Hybrid dendrimers linear polymers}

These are hybrids (block or graft polymers) of dendritic and linear polymers and having properties of both. 


\section{Amphiphilic dendrimers}

They are built with two segregated chains of which one half is electron donating and other half is electron withdrawing.

\section{Micellar dendrimers}

These are unicellular micelles of water soluble and hyper branched polyphenylenes.

\section{Multiple antigen peptide dendrimers}

It is a Dendron like molecular construct upon a polylysine skeleton. Lysine with its alkyl amino side chain serves as a good for the introduction of monomer numerous of branching points. This dendrimer was type of introduce by J. P. Tam in 1988, predominatly found its biological applications, e.g. and diagnostic vaccine research.

\section{Frechet-type dendrimers}

It is a more of dendrimer recent type developed by Hawker and Frechet based on poly benzyl ether hyper branched skeleton. These dendrimers usually have carboxylic acid groups as surface groups, serving as a good anchoring point for further surface functionlisation and as polar surface groups increase solubility of hydrophobic to this dendrimer type in polar solvents or aqueous media.

\section{Solubility Enhancement of Poorly Soluble Drugs}

Erythromycin (EM) and tobramycin (TOB) are well-known and widely used antibiotics, belonging to different therapeutic groups. Moreover, they possess different solubility: EM is slightly soluble and TOB is freely soluble in water. PAMAM dendrimers enhanced the pharmacological activity of antifungal drugs by increasing their solubility. PAMAM dendrimers significantly increased the aqueous solubility of EM, despite the increase in the solubility, there was only slight influence on the antibacterial activity of EM and also found that there was no influence of PAMAM on the antibacterial activity of hydrophilic TOB.

\section{Increases Phagocytosis}

Pneumococcal virulence factors common to all serotypes, such as choline-binding proteins (CBPs), are promising therapeutic targets in pneumococcal infections. Pneumococcal cultures were exposed to dendrimers containing choline end groups or amino groups as controls, either from the beginning of bacterial growth or at the late exponential phase. Inhibiting CBPs by micro molar concentrations of a choline dendrimer caused the formation of long pneumococcal chains that were readily phagocytosed by microglia. Long bacteria-dendrimer co-incubation resulted in a higher bacterial uptake than short coincubation. Multivalent dendrimers containing choline end groups are promising antimicrobial agents for the management of pneumococcal diseases.

\section{Permeability of Bacterial Cell Membranes}

To confirm that bacterial cell membranes were disrupted in the presence of PPI dendrimers and AMX, they are treated with propidium iodide (PI). This dye is commonly used for discriminating dead from living cells because it can enter only damaged cells with permeable membranes. PPI dendrimers penetrate through the cell wall more easily than glycodendrimers. Chen et al. showed that bacterial membranes have lower permeabilities of the larger dendrimer analogues. Destroying the cell membranes of microorganisms directly, or disrupting multivalent binding interactions between microorganisms and host cells, are the primary mechanisms of antimicrobial action by dendrimers. Cationic dendrimers act via initial electrostatic attraction to the negatively charged bacterium followed by membrane and peptidoglycan disruption. Amoxicillin inhibits bacterial cell wall synthesis so it facilitates contact of the dendrimer with the cell membrane.

\section{Dendrimeric Anti-microbial Peptide}

Antimicrobial drug resistance is a major human health threat. To tackle this problem, Peptide-based dendrimers can be designed to have higher potency than natural antimicrobial peptides with similar chemical structure but varying potency in terms of minimum inhibitory concentration were designed and at the same time they can evade the bacterial defense system.

Therapeutic macromolecules including dendrimers-based drug delivery systems exploit the pathophysiological patterns of solid tumors, particularly their leaky vasculature, to preferentially extravasate and accumulate in tumor tissue in a process known as the enhanced permeability and retention (EPR) effect. The amount of dendrimers-based drug delivery systems that accumulates in tumor tissue is influenced by their size, molecular weight, and surface charge, which affect their residence time in the systemic circulation, transport across the endothelial barrier, and nonspecific recognition and uptake by RES. El-Sayed et al. studied the effect of size, molecular weight, and surface charge on the permeability of fluorescently labelled PAMAM$\mathrm{NH}_{2}$ (G0-G4) dendrimers across epithelial and endothelial barriers. The increase in dendrimers size/molecular weight results in a corresponding exponential increase in their extravasation time $(\tau)$ across the microvascular endothelium of the cremaster muscle preparation of Syrian hamsters. Later Kobayashi et al. studied the bio-distribution of Gadolinium-functionalized G2- $\mathrm{NH}_{2}$ to $\mathrm{G} 10-\mathrm{NH}_{2}$ conjugates which showed the influence of dendrimers size/hydrodynamic volume on their transport across the microvascular endothelium in vivo. Cationic dendrimers show high nonspecific uptake by the RES particularly in the liver and lungs, which reduces their accumulation in tumor tissue. Upon comparing the biodistribution of cationic G5- $\mathrm{NH}_{2}$ dendrimers and their neutral counterparts prepared by partial or full acetylation of the surface amine groups in nude mice bearing melanoma and prostate tumors, it showed that both dendrimers displayed a similar distribution profile to all major organs within $1 \mathrm{~h}$ after dendrimers injection with particularly high accumulation in the lungs, kidneys, and liver. While the cationic and neutral dendrimers displayed similar biodistribution profiles, cationic dendrimers showed higher net accumulation in each organ due to their favorable electrostatic interaction with the negatively charged epithelial and endothelial cell surface. It is interesting to note that all polylysines, anionic PAMAM-COOH dendrimers, and polyester dendrimers exhibit high distribution to the liver and quick elimination into the urine. This biodistribution profile can be attributed to the dendrimer's small hydrody-namic volumes, which results in less than $5 \%$ of the initial dose remaining in the systemic circulation 24 $\mathrm{h}$ after administration. Attachment of PEG arms to the dendrimer surface increases their size and molecular weight, thus reducing their systemic clearance and improving their biocompatibility. Specifically, attachment of PEG chains with molecular weight up to $20 \mathrm{kDa}$ to the dendrimer's surface groups increases their plasma half-life to $50 \mathrm{~h}$ for G3 polyester dendrimers, $75.4 \mathrm{~h}$ for polylysine dendrimers, and $100 \mathrm{~h}$ for triazine dendrimers. Bhadra et al. showed that the attachment of PEG $(5 \mathrm{kDa})$ chains to $25 \%$ of the surface groups of $\mathrm{G} 4-\mathrm{NH}_{2}$ dendrimers results in a 3-fold reduction in their hemolytic activity compared to the parent dendrimers.. These studies clearly indicate the positive effect of 
surface PEGylation of PAMAM dendrimers by enhancing their plasma residence time and reducing their nonspecific toxicity.

Cationic antimicrobial peptides constitute an important component of the innate immunity against microbial infections $(1 \pm 6)$. Recently there is renewed interest in developing novel approaches for designing peptide-based antibiotics manifested by killing mechanisms that are less likely than conventional antibiotics to develop multidrug resistance $(7 \pm 12)$. Design elements desirable for therapeutics include activity under physiological conditions (100 $\pm 150 \mathrm{mM}$ or high-salt conditions), low toxicity and proteolytic stability. They have designed antimicrobial peptides with unusual structural architectures using rigid scaffoldings such as cyclic peptides highly constrained with a cystineknot motif on two or three b strands $(10 \pm 12)]$ to cluster hydrophobic and charge regions that produce amphipathic structures important for antimicrobial activity. Furthermore, these constraints confer metabolic stability, and impart membranolytic selectivity that minimizes toxicity. Another approach for designing antimicrobial peptides is based on their mechanisms of action. Pathogen-associated motifs include various microbial cell-wall components such as lipopolysaccharide (LPS), peptidoglycans, teichoic acids, mannans, N-formyl peptides, and lipidated peptides. Some well-studied motif-recognizing proteins include LPS-binding protein, soluble and membrane-anchored CD14 and Toll-like LPS receptors as well as mannose-binding protein and the receptors for mannans and manoproteins $(16 \pm 18)$. Cationic antimicrobial peptides may have also evolved to recognize PAMPs on microbial surfaces. They often possess a broad spectrum of antimicrobial activities against bacteria, fungi or viruses through mechanisms that generally involve the disruption of microbial envelopes.

\section{Membrane Disruption Dendrimer Peptide}

Many antimicrobial peptides (AMPs) act by disrupting microbial membranes. These include linear a-helical amphipathic peptides, cyclic peptides and peptoids, foldamers, various amide oligomers, and multivalent lysine dendrimers appended with linear AMPs of various lengths. In all of these cases the multiple positive charges necessary for membrane disruption are brought about by the side chains of basic amino acids. The discovery of antimicrobial peptide dendrimers such as $\mathrm{H} 1$ and bH1 in which positive charges are provided by the multiple amino termini at the dendrimer periphery. This new type of AMP acts as membrane disrupting agent and shows remarkably low haemolytic activity. Several of the dendrimers also showed good activities against $E$. coli and $P$. aeruginosa, in particular the Dapbranched analogs $\mathrm{bH} 1$ and $\mathrm{bH} 2$, which were also more than the lysine branched dendrimers. The peptide dendrimers were generally less active on Gram negative bacteria, which together with the activity of the dendrimer stereoisomers and the requirement for a combination of cationic charges and hydrophobic residues pointed towards membrane disruption as a possible mechanism of action.

\section{Nano conjugates for Anti-bacterial Therapy}

The elevated altitudes of control possible over the dendrimers architectural design are their shape, size, surface functionality and their branching length/density. The bioactive agents may be condensed into the interior of the dendrimers or physically adsorbed/chemically attached onto the dendrimer surface. It has been shown that Modified dendrimers act as nano-drugs against bacteria, viruses and tumors. PAMAM dendrimers are potential carriers for drug delivery due to their unique structure. Penicillin V was used as a model carboxylic group containing drug to conjugate with full- and half-generation.
A drug carrying a carboxylic group (e.g. penicillin V) was coupled to star polymer via amide and ester bonds. A single-strain bacterium, Staphylococcus aureus, was grown up for penicillin-conjugated PEGPAMAM star polymer activity test. The bioavailability of modified penicillin after the ester bond was cleaved.

\section{Dual Acting Anti-bacterial Agents}

Besides acting as antimicrobial compounds, dendrimers can be considered as agents that improve the therapeutic effectiveness of existing antibiotics. A new approach using amoxicillin (AMX) against reference strains of common Gram-negative pathogens, alone and in combination with poly (propylene imine) (PPI) dendrimers, or derivatives. The PPI dendrimers significantly enhance the antibacterial effect of amoxicillin alone, allowing antibiotic doses to be reduced. It is important to reduce doses of amoxicillin because it leads to the development of bacterial resistance and environmental pollution.

\section{Against Resistant Strain}

PEGylated poly (propylene imine) dendritic architecture was loaded with Ciprofloxacin and targeted to the resistants produce strains of Staphylococcus aureus and Cryptococcus pneumonia. The Ciprofloxacin loaded dendrimer has significant antibacterial activity than the plain PPI dendrimer, but standard drug was not shown zone of inhibition upon both microorganisms. The antibacterial activity of system is also relatively safer and hold potential to deliver some other drugs also.

\section{Anti-bacterial Activity}

Owing to antibacterial properties of dendrimers, they can be used as alternative water and waste water disinfection with the minimum adverse side effects. It can be used to evaluate the antibacterial activity of predominant bacteria in drinking water resources. Therefore, it is possible to use these nano dendrimers as a safe and effective material for water disinfection in the future. Antimicrobial is a substance that kills or inhibits the growth of microorganisms (Merriam-Webster Online Dictionary, 2009) such as bacteria, fungi, or protozoans. Antimicrobial drugs either kill microbes (microbiocidal) or prevent the growth of microbes (microbiostatic). Disinfectants are antimicrobial substances used on non-living objects or outside the body (Wikipedia, 2011). Antibacterial and antimicrobial are two similar concepts and sometimes they are used interchangeably. Many antibacterial products for cleaning and hand washing are sold today.

PEGylated poly(propylene imine) dendritic architecture was synthesized and loaded with Levofloxacin and targeted to the resistant producing strains of Klebsiella pneumoniae and E. coli. The Levofloxacin loaded dendrimer has significant antibacterial activity than the plain drug and control. Antibacterial activity of synthesized system is also relatively safer and hold potential to deliver some other drugs also.

\section{Antibacterial agents work by}

\section{Interference with cell wall synthesis}

Lactams: penicillins, cephalosporins, carbapenems, monobactams; Glycopeptides: vancomycin, teicoplanin

\section{Protein synthesis inhibition}

- Bind to 50 S ribosomal subunit: macrolides, chloramphenicol, clindamycin, quinupristin-dalfopristin, linezolid 
- Bind to 30 S ribosomal subunit: aminoglycosides, tetracyclines

- $\quad$ Bind to bacterial isoleucyl-tRNA synthetase: mupirocin

\section{Interference with nucleic acid synthesis}

- Inhibit DNA synthesis: fluoroquinolones

- Inhibit RNA synthesis: rifampin

- Inhibition of metabolic pathway: sulfonamides, folic acid analogues

- Disruption of bacterial membrane structure: polymyxins, daptomycin

High molecular surface functional group concentration of dendrimers can dominate antibacterial properties to the interacting molecule. If the end or surface groups of dendrimers are functionalized with biologically active antimicrobial groups, might expect an increase in antimicrobial activity of the dendrimers. Biocides immobilized on dendrimers can be more effective if the target sites are cell walls and/ or membranes. It has been shown that small quaternary ammonium compounds exert their antimicrobial action by disrupting and disintegrating the cell membrane. Dendrimer biocides may be very beneficial in terms of activity, localization in specific organs, reduced toxicity, and increased duration of action (Donaruma, 1978, 1980). As the bacteria are negatively charged and dendrimer biocides have high positive charge density, electrostatic interactions bring them into contact with each other. High concentrations of dendrimer biocides can lead to complete disintegration of the bacterial membrane causing to a bactericidal effect.

By adding water soluble functional end groups to dendrimers, water soluble dendrimers are obtained. When these dendrimers interacted with bacteriostatic weak water soluble or insoluble antibiotics, the antibacterial properties antibiotics can be altered, especially improved. Microbiological studies of the quinolones (nadifloxacin and prulifloxacin) showed that strong antimicrobial activities of nadifloxacin and prolifloxacin were still significantly increased in the presence of PAMAM dendrimers and also their water solubility increased. Poly (amido amine) PAMAM (e.g, the generation 3 (G3) PAMAM) dendrimers are the most extensively studied dendrimers. PAMAM dendrimers with a wide variety of functional groups at the periphery are commercially available. Some of the dendrimers having terminal amino groups are shown as they are having low toxicity to eukaryotic cells. Modification of the amino groups of the PAMAM dendrimers with poly (ethylene glycol) (PEG) or lauroly chains further improves the biocompatibility.

There is a significant global need for new antibacterial and alternative mechanisms of action given the rise in resistance among bacteria. Of the various known antibacterial agent classes, amphiphilic compounds act through perturbation and disruption of the prokaryotic membrane. It has been hypothesized that amphiphilic anionic dendrimers may exhibit antibacterial activity with minimal eukaryotic cell cytotoxicity, since dendrimers with terminal anionic charges are generally noncytotoxic and have low toxicity in zebrafish whole animal development studies. On the other hand, cationic dendrimers, some of which have antibacterial properties if the positive charge is properly shielded, have repeatedly shown cytotoxicity against a variety of eukaryotic cell lines. Metal containing dendritic nanoparticles called as the Metallodendrimers. They are incorporated with metal atoms. Silver containing compounds and materials have been routinely used to prevent attack of a broad spectrum of microorganisms on prostheses, catheters, vascular grafts, human skin, also used in medicine to reduce infection in burnt treatment, arthroplasty. However they exhibit low toxicity to mammalian cells. The antibacterial activity of silver nanoparticles increases with the increase of concentration of the active agent. Dispersion of organic/inorganic hybrid materials could be utilized to form regular thin film coatings with antibacterial effects by using dendritic-polymer templates. The antibacterial activity of the coating films based on the hyper branched core/shell type hybrids and closely associated with the silver ions release of the films. Nano-scaled silver may (1) release silver ions and generate reactive oxygen species (ROS); (2) interact with membrane proteins affecting their correct function; (3) accumulate in the cell membrane affecting membrane permeability; and (4) enter into the cell where it can generate ROS release silver ions, and affect DNA. Generated ROS may also affect DNA, cell membrane, and membrane proteins, and silver ion release will likely affect DNA and membrane proteins.

To inhibit the binding of the $B_{5}$ subunit to cell surface GM1 Schengrund group has developed oligosaccharide functionalized dendrimers. The dendritic cores used were tetra ( $1^{\text {st }}$ generation) $(\mathbf{1})$ and octa (propylene imine) ( $2^{\text {nd }}$ generation) (2) dendrimers and the $1^{\text {st }}$ generation of Startburst ${ }^{\mathrm{TM}}$ (PAMAM) (3). Pieters have prepared dendrimers with a significant rigid backbone using 3,5-bis(2aminoethoxy)benzoic acid as repeating unit. Dendrimers of first G1 (4), second G2 (5), and third G3 (6) generations presenting 2, 4, and 8 lac-tose as sugars. The affinity of these compounds for cholera toxin B subunit was measured using fluorescence spectroscopy. In this study was also pointed out the importance of the size and shape of the spacer used to attach the carbohy-drate moiety to the dendritic core. Bernardi and Pieters groups have prepared a dendrimer using the same dendritic core described above functionalized with a GM1 mimic. This system was tested for CTB binding using two techniques, SPR analysis and ELISA assays. ELISA was performed with the aim to evaluate the activity of this octavalent com-pound. The ELISA wells were coated with the ganglioside.

One of the more remarkable example in the rational design of multivalent systems for the inhibition of SLTs was described by named STARFISH designed by Bundle. Nishikawa have studied the inhibition of infection by shiga toxin-producing 0157:H7 Using carbosilane dendrimers functionalized with the same Gb3 trisaccharide. These dendritic structures named SUPER TWIG are constituted by a core of silicon-carbon bonds that are biologically inert. David have used polycationic amine-terminated poly(amidoamine) (PAMAM) dendrimers as endotoxin sponge for the therapy of Gram-negative bacterial sepsis. By using BODIPY cadaverine as displacement probe the affinity of these dendrimers for LPS was evaluated using a highthroughput fluorescence displacement method. assays of nitric oxide release in LPS-stimulated murine macrophage were used to analyze the dendrimer activity. To neutralize endotoxins amphiphilic character of these dendrimers partially functionalized with alkyl groups. Lindhorst have developed carbohydrate multivalent systems to inhibit the adhesion of mediated by 1 fimbriae and were made as potential inhibitors based on multivalent systems containing 3 (7), 4 (8), 6 (9), and 8 (10) mannopyranosides attached to the scaffold through a thiourea linkage were prepared and their activities were tested. The studies revealed important issues concerning the importance of the mannose orientation on these types of multivalent systems, affinities of these were strongly dependent on the distance between mannose residues; a large distance $(>20 \mathrm{~nm})$. 
Pieters have developed small multivalent systems, glycodendrimers and glycopolymers to inhibit the adhesion of type 1 fimbriateduropathogenic. The activity of these was tested using zone of inhibition growth with bacteria on a solid agar medium. This dendronized system, when it was doped with silver, a clear inhibition was observed. Urbanczyk-Lipkowska have prepared two type of dendrimers based into amino acids (Lysine) to inhibit infection by and growth was evaluated by Minimal inhibitory concentrations (MIC). Cooper have developed quaternary ammonium functionalized poly(propylene imine) dendrimers (12) as antimicrobials. The biocide activity was evaluated using a biolumines-cence method. Magnusson in 1997 prepared a small tetravalent galabiose system to inhibit the hemagglutination by at nanomolar concentration. Pieters described the preparation of galabiose dendrimers and their inhibition activities against. To study the inhibition of hemagglutination of human erythrocytes induced by two subtypes of $\left(\mathrm{P}_{N}\right.$ and $\left.\mathrm{P}_{\mathrm{O}}\right)$ was performed. The MIC (minimal inhibitory concentration) required for complete inhibition of the agglutination process were measured. The dendrimers described by Cooper and Ur-banczyk-Lipkowska for were also tested against a Gram-positive bacteria NCTC 4163. Davis have developed a newtype of multivalent systems named glycodendriproteins consisting in the functionalization of proteins with glycodendrons and are mimicking glycoproteins avoided the problem of glycoforms. They were tested as potential inhibitors of co-aggregation of and UrbanczykLipkowska where dendrimers are used as anti-infective agents in a infection process. These authors have developed a low molecular mass lysine dendrimer with antimicrobial activity functionalized at the surface by arginin residues. Prusiner have demonstrated that highly branched polyamidoamine PAMAM dendrimers were able to eliminate $\mathrm{PrP}^{\mathrm{Sc}}$ in a very efficient way. It seems that the presence of den-drimers make fibrils sensitive to protease Kdegradation. Very recently, Cladera have studied the aggregation of the Alzheimer amyloid peptide A 1-28 and human prion protein PrP185-208 in the presence of PAMAM dendrimers. Higher generations of PAMAM G3-G5 led to a smaller amount of fibrils formed. $1 \mathrm{M}$ of PAMAM G5 was enough to inhibit the fibril formation. Heegaard have used a guanidinium modified dendrimer (15) based on the second generation of poly (propyl-ene imine) (PPI) (2) to destabilized the fibril formation of a peptide fragment of the PrP. Most of the work concerning dendrimers is oriented to developing these microbicides of topical use. HIV inhibition activities and cytotoxicities of these dendrimers were evaluated using CEM-SS cells (human T4-lymphoblastoid cell line) as model. One of the promising compounds developed against HSV is a sulphated polylysine dendrimer named SPL2999. An evolution of this compound is the dendrimer. This dendrimer have been tested as a microbicide candidate against genital herpes in mouse and guinea pig models. A new study concerning the antiviral efficacy, mechanism of action, and toxicity. SPL7013 inhibited virus internalization of both HSV-1 and HSV-2. Also, SPL7013 showed post-exposure activity on HSV infection indicating a therapeutic activity with a $\mathrm{pH}$ independent activity. Again, SPL7013 has been demonstrated as a promising microbicide candidate in STIs.

Whitesides described in 1999 a pioneer work in the preparation of multivalent systems based on polyacrylamide functionalized with -sialoside groups. This glycopolymer strongly inhibited the agglutination of erythrocytes by influenza virus. Baker developed systems based on PAMAM as dendritic core functionalized with sialic acid as described in the original papers and in a recent review. These dendritic structures could inhibit the infection process in a DC-SIGN dependent manner. Our strategy was oriented to inhibit the entry of
Ebola virus blocking the DC-SIGN lectin, a receptor that was described as one of the potential gate of entrance for this virus. These types of glycostructures were able to block the DC-SIGN receptor at the cell surface inhibiting the entrance of the pathogen and therefore, they could be used as microbicides. Most of the structures used as antiinfective agents are based on poly(amido amine) (PAMAM) and poly(propylene imine) (PPI) dendrimers. These are two well-known structures and both of them commercially available up to generation 5 at least. Chemical manipulation of the dendritic surface are produced a large number of new structures with interesting antimicrobial. Changes of the functional groups at the surface or even the chemical structure of the core can be manipulated to achieve low toxicities. The dendrimers and dendritic polymers have to play a key role in the nanoscience field. They are very flexible structures to be easily manipulated and modulated by chemists to achieve new properties and pursue future applications in biomedical sciences. In fact, the knowledge provided by all the research summarized above, establishes the bases for new accomplishments in the area of anti-infective agents with a broad spectrum of applications. Having a compound in clinical Phase I means a real step towards achieving the first milestone. We hope that in the next few decades, we could have derivatized dendritic compounds to be used as pharmaceuticals.

\section{Conclusion}

Around the world in many healthcare facilities, bacterial pathogens that express multiple resistance mechanisms are becoming the norm, increasing both human morbidity and financial costs and complicating treatment. To eliminate most intracellular bacteria such us or till now, no antibiotic therapy has been reported. To reduce the disease relapses down to 5-15\% still more, a prolonged exposure to combined antibiotics is required. Keeping view in this, drug delivery scientists are searching for the ideal nanovehicle for the ideal nanodrug delivery system; one that would dramatically improve in the drug absorption, reduce drug dosage so that the patient can take a smaller dose, and yet have the same benefit, deliver the drug to the right place in the living system, limit or eliminate side effects and increase the local concentration of the drug at the favorite site. Compared with other polymeric particles, colloidal carriers mainly nanoparticles, have appeared more recently as attractive carriers for the delivery of drugs to infected cells. Synthetic biodegradable and biocompatible polymers have been shown to be effective for encapsulating a great variety of antibiotics. Phagocytosis is enhanced powerfully by these polymeric particles and are suitable for antibacterial agents for intracellular delivery. There is no doubt that nanoparticle-based drug delivery systems will continue to improve treatment to bacterial infections due to the incessant attempts in this field.

\section{References}

1. Brown GD, Taylor PR, Reid DM, Willment JA, Williams, DL (2002) B-(1,3)-Dglucan modulates DNA binding of macrophages. J Exp Med 196: 407-18.

2. Bakker-Woudenberg IAJM (1995) Delivery of antimicrobials to infected tissue macrophages. Adv. Drug Deliv Rev 17: 5-20.

3. Coates A, Hu Y, Bax R, Page C (2002) The future challenges facing the development of new antimicrobial drugs. Nat Rev Drug Discov 1: 895-910.

4. Walker CB (1996) Selected antimicrobial agents: mechanisms of action, side effects and drug interactions. Periodontol 2000 10: 12-28.

5. Zhang L, Gu FX, Chan JM, Wang AZ, Langer RS, et al. (2008) Nanoparticles in medicine: therapeutic applications and developments. Clin Pharmacol Ther 83: $761-769$

6. Davis ME, Chen ZG, Shin DM (2008) Nanoparticle therapeutics: an emerging treatment modality for cancer. Nat Rev Drug Discov 7: 771-782. 
7. Peer D, Karp JM, Hong S, Farokhzad OC Margalit R, et al. (2007) Nanocarriers as an emerging platform for cancer therapy. Nat Nanotechnol 2: 751-760.

8. Wagner V, Dullaart A, Bock AK, Zweck A (2006) The emerging nanomedicine landscape. Nat Biotechnol 24: 1211-1217.

9. Zhang L, Granick S (2006) How to stabilize phospholipid liposomes (using nanoparticles). Nano Lett 6: 694-698.

10. Farokhzad OC, Langer $R$ (2006) Nanomedicine: developing smarter therapeutic and diagnostic modalities. Adv Drug Deliv Rev 58: 1456-1459.

11. Lian T, Ho RJ (2001) Trends and developments in liposome drug delivery systems. J Pharm Sci 90: 667-680.

12. Hiemenz JW, Walsh TJ (1996) Lipid formulations of amphotericin B: recent progress and future directions. Clin Infect Dis 22 Suppl 2: S133-144.

13. Adler-Moore JP, Proffitt RT (1993) Development, characterization, efficacy and mode of action of AmBisome, a unilamella liposomal formulation of amphotericin B. J Liposome Res 3: 429-50.

14. Adler-Moore JP, Proffitt RT (1998) AmBisome: long circulating formulation of Amphotericin B. Springer-Verlag, Berlin 5: 185-206.

15. Adler-Moore J, Proffitt RT (2002) AmBisome: liposomal formulation, structure mechanism of action and pre-clinical experience. J Antimicrob Chemother 49 Suppl 1: 21-30

16. Alipour M, Halwani M, Omri A, Suntres ZE (2008) Antimicrobial effectiveness of liposomal polymyxin B against resistant Gram-negative bacterial strains. Int J Pharm 355: 293-298.

17. Fischbach MA, Walsh CT (2009) Antibiotics for emerging pathogens. Science 325: 1089-1093.

18. Schumacher I, Margalit R (1997) Liposome-encapsulated ampicillin physicochemical and antibacterial properties. J Pharm Sci 86: 635-641.

19. Kim HJ, Jones MN (2004) The delivery of benzyl penicillin to Staphylococcus aureus biofilms by use of liposomes. J Liposome Res 14: 123-139.

20. Magallanes M, Dijkstra J, Fierer J (1993) Liposome-incorporated ciprofloxacin in treatment of murine salmonellosis. Antimicrob Agents Chemother 37: 22932297.

21. Venugopal J, Prabhakaran MP, Low S, Choon AT, Deepika G, et al. (2009) Continuous nanostructures for the controlled release of drugs. Curr Pharm Des 15: $1799-1808$.

22. Langer R, Folkman J (1976) Polymers for the sustained release of proteins and other macromolecules. Nature 263: 797-800.

23. Gref R, Minamitake Y, Peracchia MT, Trubetskoy V, Torchilin V, et al. (1994) Biodegradable long-circulating polymeric nanospheres. Science 263: 1600-1603.

24. Abeylath SC, Turos E, Dickey S, Lim DV (2008) Glyconanobiotics: Nove carbohydrated nanoparticle antibiotics for MRSA and Bacillus anthracis. Bioorg Med Chem 16: 2412-2418.

25. Espuelas MS, Legrand P, Loiseau PM, Bories C, Barratt G, et al. (2002) In vitro antileishmanial activity of amphotericin B loaded in poly(epsilon-caprolactone) nanospheres. J Drug Target 10: 593-599.

26. Domb AJ (1993) Liposphere parenteral delivery system. Proc Intern Symp Control Rel Bioact Mater 20: 346-7.

27. Müller RH, Radtke M, Wissing SA (2002) Solid lipid nanoparticles (SLN) and nanostructured lipid carriers (NLC) in cosmetic and dermatological preparations. Adv Drug Deliv Rev 54 Suppl 1: S131-155.

28. Wissing SA, Müller RH (2003) Cosmetic applications for solid lipid nanoparticles (SLN). Int J Pharm 254: 65-68.

29. Müller RH, Mäder K, Gohla S (2000) Solid lipid nanoparticles (SLN) for controlled drug delivery - a review of the state of the art. Eur J Pharm Biopharm 50: 161-177.

30. Wissing S, Lippacher A, Müller R (2001) Investigations on the occlusive properties of solid lipid nanoparticles (SLN). J Cosmet Sci 52: 313-324.

31. Gupta AK, Cooper EA (2008) Update in antifungal therapy of dermatophytosis Mycopathologia 166: 353-367.

32. Bosman AW, Janssen HM, Meijer EW (1999) About dendrimers: Structure, physical properties, and applications. Chem Rev 99: 1665-1688.
33. Gillies ER, Fréchet JM (2005) Dendrimers and dendritic polymers in drug delivery. Drug Discov Today 10: 35-43.

34. Florence AT (2005) Dendrimers: a versatile targeting platform. Adv Drug Deliv Rev 57: 2104-5.

35. Chen CZ, Cooper SL (2002) Interactions between dendrimer biocides and bacterial membranes. Biomaterials 23: 3359-3368.

36. Balogh L, Swanson DR, Tomalia DA, Hagnauer GL, McManus et al. (2001) Dendrimer-Silver Complexes and Nanocompo-sites as Antimicrobial Agents. Nano Lett 1: 18-21.

37. Ma M, Cheng $Y, X u Z, X u ~ P, Q u ~ H$, et al. (2007) Evaluation of polyamidoamine (PAMAM) dendrimers as drug carriers of anti-bacterial drugs using sulfamethoxazole (SMZ) as a model drug. Eur J Med Chem 42: 93-98.

38. Omri A, Suntres ZE, Shek PN (2002) Enhanced activity of liposomal polymyxin $B$ against $P$ seudomonas aeruginosa in a rat model of lung infection. Biochem Pharmacol 64: 1407-1413.

39. Mimoso IM, Francisco APG, Cruz MEM (1997) Liposomal formulation of netilmicin. Int J Pharm 147: 109-17.

40. Schiffelers R, Storm G, Bakker-Woudenberg I (2001) Liposome-encapsulated aminoglycosides in pre-clinical and clinical studies. J Antimicrob Chemother 48: 333-344

41. Berton M, Turelli P, Trono D, Stein CA, Allémann E, et al. (2001) Inhibition of HIV-1 in cell culture by oligonucleotide-loaded nanoparticles. Pharm Res 18 1096-1101.

42. Shah LK, Amiji MM (2006) Intracellular delivery of saquinavir in biodegradable polymeric nanoparticles for HIVIAIDS. Pharm Res 23: 2638-2645

43. Pandey R, Khuller GK (2006) Oral nanoparticle-based antituberculosis drug delivery to the brain in an experimental model. J Antimicrob Chemother 57 $1146-1152$.

44. Souto EB, Müller RH (2005) SLN and NLC for topical delivery of ketoconazole. J Microencapsul 22: 501-510.

45. Cheng Y, Qu H, Ma M, Xu Z, Xu P, et al. (2007) Polyamidoamine (PAMAM) dendrimers as biocompatible carriers of quinolone antimicrobials: an in vitro study. Eur J Med Chem 42: 1032-1038.

46. Bhadra D, Bhadra S, Jain NK (2005) Pegylated lysine based copolymeric dendritic micelles for solubilization and delivery of artemether. J Pharm Pharm Sci 8: 467-482.

47. Ahmad Z, Pandey R, Sharma S, Khuller GK (2006) Alginate nanoparticles as antituberculosis drug carriers: formulation development, pharmacokinetics and therapeutic potential. Indian J Chest Dis Allied Sci 48: 171-176.

48. Bala T, Armstrong G, Laffir F, Thornton $R$ (2011) Titania-silver and aluminasilver composite nanoparticles: Novel, versatile synthesis, reaction mechanism and potential antimicrobial application. J Colloid Interface Sci 356: 395-403.

49. Beaulac C, Clement-Major S, Hawari J, Legace J (1996) Eradication of mucoid Pseudomonas aeruginosa with fluid liposome-encapsulated tobramycin in an animal model of chronic pulmonary infection. AntimicrobAgents Chemother 40 665-669.

50. Berkowitz FE (1995) Antibiotic resistance in bacteria. South Med J 88: 797-804

51. Bhadra D, Bhadra S, Jain NK (2005) PEGylated peptide based dendritic nanoparticulate systems for delivery of artemether. J Drug Del Sci Tech 15 $65-73$.

52. Cavalli R, Gasco MR, Chetoni P, Burgalassi S, Saettone MF (2002) Solid lipid nanoparticles (SLN) as ocular delivery system for tobramycin. Int J Pharm 238 : 241-245.

53. Chakraborty SP, Sahu SK, Mahapatra SK, Santra S, Bal M, et al. (2010) Nanoconjugated vancomycin: new opportunities for the development of antiVRSA agents. Nanotechnology 21: 105103.

54. Chan JM, Zhang L, Yuet KP, Liao G, Rhee JW, et al. (2009) PLGA-lecithin-PEG core-shell nanoparticles for controlled drug delivery. Biomaterials 30: 16271634.

55. Chopra I (2007) The increasing use of silver-based products as antimicrobial agents: a useful development or a cause for concern? J Antimicrob Chemothe 59: 587-590.

56. Chung YC, Wang HL, Chen YM, Li SL (2003) Effect of abiotic factors on the 
Citation: Karthikeyan R, Kumar PV, Koushik OS (2016) Dendrimeric Biocides - A Tool for Effective Antimicrobial Therapy. J Nanomed Nanotechnol 7: 359. doi:10.4172/2157-7439.1000359

antibacterial activity of chitosan against waterborne pathogens. Bioresour Technol 88: 179-184

57. Cohen ML (2000) Changing patterns of infectious disease. Nature 406: 762-767.

58. Espuelas MS, Legrand P, Campanero MA, Appel M, Chéron M, et al. (2003) Polymeric carriers for amphotericin $\mathrm{B}$ : in vitro activity, toxicity and therapeutic efficacy against systemic candidiasis in neutropenic mice. J Antimicrob Chemother 52: 419-427.

59. Esteban TL, Malpartida F (2009) Antibacterial and antifungal activity of a sodalime glass containing copper nanoparticles. Nanotechnology 20: 505-701.

60. Ferrari M (2005) Cancer nanotechnology: opportunities and challenges. Nat Rev Cancer 5: 161-171.

61. Gold HS, Moellering RC Jr (1996) Antimicrobial-drug resistance. N Engl J Med 335: $1445-1453$

62. Hetrick EM, Shin JH, Paul HS, Schoenfisch MH (2009) Anti-biofilm efficacy of nitric oxide-releasing silica nanoparticles. Biomaterials 30: 2782-2789.

63. Jain KK (2007) Applications of nanobiotechnology in clinical diagnostics. Clin Chem 53: 2002-2009.
64. Kaur CD, Nahar M, Jain NK (2008) Lymphatic targeting of zidovudine using surface-engineered liposomes. J Drug Target 16: 798-805.

65. Taylor PW, Stapleton PD, Paul Luzio J (2002) New ways to treat bacteria infections. Drug Discov Today 7: 1086-1091.

66. Taubes G (2008) The bacteria fight back. Science 321: 356-361.

67. Weinberg ED1 (1999) Iron loading and disease surveillance. Emerg Infect Dis 5: 346-352.

68. Yang J, Lee J, Kang J, Lee K, Suh JS, et al. (2008) Hollow silica nanocontainers as drug delivery vehicles. Langmuir 24: 3417-3421.

69. Zhang L, Pornpattananangku D, Hu CM, Huang CM (2010) Development of nanoparticles for antimicrobial drug delivery. Curr Med Chem 17: 585-594.

70. Mizrahi A, Ben-Ner E, Katz MJ, Kedem K, Glusman JG, et al. (2000) Comparative analysis of dendritic architecture of identified neurons using the Hausdorff distance metric. J Comp Neurol 422: 415-428.

71. Smith PB, Martin SJ, Hall MJ, Tomalia DA (1987) A Characterization of the Structure and Synthetic Reactions of Polyamidoamine "STARBURST" Polymers. Munich 3: 357-385. 